\title{
Hydraulique des ouvrages de franchissement des vallées fluviales
}

\author{
The hydraulics of structures \\ for crossing river valleys
}

\author{
G. Nicollet \\ Electricité de France \\ Direction des Etudes et Recherches - Chatou \\ Laboratoire National d'Hydraulique
}

\section{Introduction}

Le franchissement d'un cours d'eau et de sa vallée par des ouvrages d'art ne se limite pas en général à la construction d'un pont enjambant le lit mineur. Les accès au pont sont bien souvent implantés dans le lit majeur, ce qui nécessite une étude globale de l'écoulement des crues exceptionnelles pour définir les caractéristiques des ouvrages.

Plusieurs disciplines sont impliquées dans l'élaboration de l'avant-projet :

- l'hydrologie pour la définition de la crue exceptionnelle type de projet;

- l'hydraulique pour la façon dont cette crue franchira les ouvrages;

- la mécanique des sols pour la reconnaissance du terrain ;

- la technique de construction proprement dite pour le calcul des structures et de leurs fondations.

Le présent document qui se limite exclusivement aux problèmes hydrauliques et à leurs conséquences, vise à fournir au projeteur de pont des méthodes simples permettant de dimensionner un ouvrage. Des exemples d'application sont fournis en annexe.

\section{Remarques :}

1) Ce document ne concerne que les ponts modernes, en béton ou métalliques, dont la section horizontale des appuis est constante sur toute la hauteur. Par exemple, il ne saurait être utilisé pour calculer les effets des anciens ponts en maçonnerie à arches multiples.

2) Une solution d'économie, parfois utilisée dans les pays peu développés, consiste à accepter que le pont et ses accès soient submergés à chaque crue importante. Dans notre pays, par contre, on cale le tablier à une cote telle qu'il soit toujours hors d'eau; c'est pourquoi le présent document n'envisage pas le cas des ponts en charge.

\section{Objectifs}

\subsection{Critères hydrauliques des ouvrages de franchissement}

Dans le cas général les ouvrages se composent :

- du pont proprement dit ;

- des accès, le plus souvent des remblais, dont le coût au mètre linéaire est nettement moindre que celui du pont.

Cette évidente considération économique conduit à minimiser l'ouverture du pont quitte à compenser son étroitesse par des ouvrages de décharge dans le lit majeur. L'ordre de grandeur habituellement adopté pour l'ouverture du pont est celui de la largeur du lit mineur du cours d'eau.

En période de crue, un pincement de l'écoulement se manifeste donc au passage des ouvrages, d'où les deux conséquence fondamentales suivantes sur le comportement hydraulique :

a) le niveau de l'eau est exhaussé en amont, c'est le phénomène de remous ;

b) l'écoulement est accéléré au niveau du rétrécissement, ce qui peut entraîner des affouillements mettant en cause la stabilité de l'ouvrage.

L'étude hydraulique d'un avant-projet consiste à chiffrer ces deux éléments, remous et profondeur d'affouillement, pour les comparer aux impératifs de sécurité et de coût. Au cas où l'on aboutit à une incompatibilité, il convient de reprendre le projet en modifiant un ou plusieurs choix jusqu'à obtenir des résultats compatibles.

\section{I.2. Articulation de l'élaboration d'un projet}

Le graphe de la figure 1 schématise les étapes successives à franchir pour aboutir à l'élaboration d'un avantprojet. Les phases représentées par des rectangles corres- 


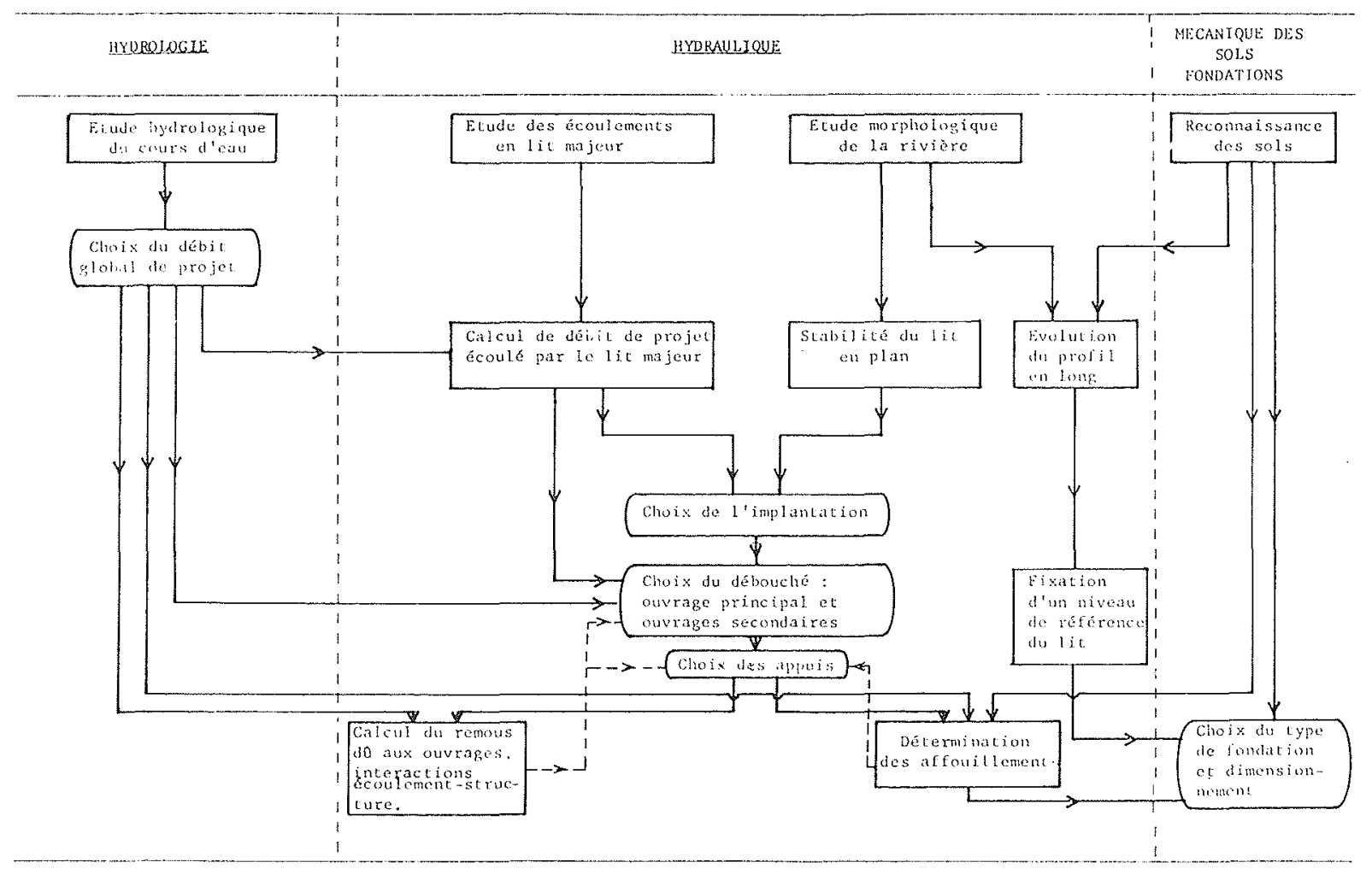

Figure 1 - Graphe sagittal de l'établissement d'un projet d'ouvrages de franchissement de vallée.

pondent à des études permettant d'acquérir des informations à partir desquelles seront effectués des choix représentés par des cadres oblongs, qui définissent des ouvrages.

A chaque étape de l'étude, les résultats doivent être confrontés aux impératifs techniques, de sécurité, d'environnement et de coût. En cas d'incompatibilité, il convient de revenir en arrière pour modifier un ou plusieurs des choix.

Les phases successives du travail sont :

\section{a) Le choix du débit de projet}

Choix basé sur une étude hydrologique de la section considérée du cours d'eau (étude qui ne sera pas envisagée ici).

\section{b) Le calcul du débit écoulé par le lit majeur pour la crue type}

Le débit global de projet ayant été fixé, il convient de s'assurer, dans le cas où il existe un lit majeur, si ce dernier est simplement capacitif ou au contraire actif pour la crue considérée. Si le lit majeur est actif, une étude de l'écoulement, basée sur les observations naturelles disponibles (submersions, vitesses), doit ètre effectuée sur modèle mathématique si les lits sont quasi rectilignes et assez bien définis, où éventuellement sur modèle physique dans les cas complexes de lit sinueux.

Le modèle mathématique sera essentiellement composé d'un programme de calcul des courbes et remous en lit composé (programme Debord, [2]).

S'il est nécessaire de recourir à un modèle physique, il est bien évident qu'il sera utilisé pour résoudre la plupart des problèmes hydrauliques du franchissement dont il est question par la suite.

Que ce soit sur modèle physique, ou sur modèle mathématique, le processus d'étude est le même. I consiste à étalonner le modèle pour des écoulements connus :

- étalonnage du lit mineur au voisinage de la limite de débordement par représentation de lignes d'eau relevées en nature ;

- étalonnage du lit majeur à partir des données naturelles recueillies lors de crues suffisamment importantes. C'est ce point qui est le plus souvent délicat à réaliser en raison des renseignements très fragmentaires de cotes et de vitesses qui peuvent actuellement être recueillis concernant des crues anciennes; à cela s'ajoute souvent une imprécision sur la topographie et l'état de la végétation au moment de ces crues. Dans de nombreux cas, on en est réduit à formuler des hypothèses sur l'écoulement des crues passées pour pouvoir étalonner le modèle.

Le modèle ainsi étalonné est utilisé pour représenter l'écoulement du débit global de la crue du projet afin de déterminer la répartition des débits entre le lit mineur et le champ d'inondation de même que la valeur des vitesses moyennes d'écoulement.

Dans l'hypothèse où le lit est suffisamment régulier au voisinage de la section du futur franchissement, l'écoulement peut être considéré comme uniforme pour la crue du projet. II est alors possible d'évaluer, rapidement, à la plume, la répartition du débit entre lit mineur et lit majeur. La méthode basée sur l'application de la loi Debord, [2], est la suivante : 
- évaluation du coefficient de Strickler mineur $K m$ pour une ligne d'eau à la limite de débordement : $K m=Q /\left(S R m^{2 / 3} J^{1 / 2}\right)$;

- évaluation du coefficient de Stickler majeur $K M$ pour la ligne d'eau extrémale de la plus grande crue connue.

On recherche $K M$ par tâtonnement pour satisfaire la loi Debord ci-dessous où $D e$ désigne la débitante équiva-

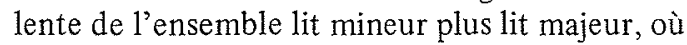

$$
\begin{gathered}
D e=Q / J^{1 / 2} \\
D e=K m A S m R m^{2 / 3}+ \\
+K M \sqrt{S^{2} M+S m S M\left(1-A^{2}\right)} \cdot R M^{2 / 3}
\end{gathered}
$$

arec

$$
\begin{array}{ll}
A=A_{0}=0,9\left(\frac{K m}{K M}\right)^{-1 / 6} \quad \text { pour } \quad r=\frac{R M}{R m} \geqslant 0,3 \\
A=\frac{1-A_{0}}{2} \cos \frac{\pi r}{0,3}+\frac{1+A_{0}}{2} \quad \text { pour } \quad r<0,3
\end{array}
$$

l'indice $m$ caractérise le lit mineur et l'indice $M$ le lit majeur.

- recherche de la cote de passage de la crue du projet par application de la loi Debord ci-dessus, ou à défaut par simple extrapolation de la loi hauteur-débit ;

- calcul de la répartition de débit pour la crue du projet :

$$
\begin{gathered}
\eta=\frac{Q m}{Q M}=\frac{K m}{K M} \frac{A S m}{\sqrt{S^{2} M+S m S M\left(1-A^{2}\right)}}\left(\frac{R m}{R M}\right)^{2 / 3} \\
Q M=Q /(1+\eta)
\end{gathered}
$$

\section{c) L'étude de la stabilité en plan du lit}

La confrontation des levés topographiques successifs (cartes d'état-major, photographies aériennes) permet de se former une opinion sur l'évolution du tracé en plan du lit. Deux cas se présentent :

- ou bien le cours d'eau a atteint un état d'équilibre avec un tracé bien fixé depuis de nombreuses décennies, auquel cas l'étude de la stabilité en plan est close,

- ou bien des évolutions se sont manifestées encore récemment, auquel cas une étude approfondie sur plan ou éventuellement sur modèle sédimentologique est nécessaire pour apprécier les risques d'évolutions futures et mettre au point des dispositifs capables de fixer les berges. Ce point sera étudié dans le chapitre des érosions.

Il est essentiel de tenir compte des travaux en cours ou des projets futurs d'aménagement du cours d'eau : qu'il s'agisse de dragages, de recalibrages, d'édification de barrages, tous travaux susceptibles de remettre en cause l'équilibre du lit et dont il sera question au chapitre "érosion".

\section{d) Le choix de l'implantation des ouvrages}

Connaissant les débits à écouler respectivement par les lits mineur et majeur et s'étant assuré de la stabilité du lit en plan, le projecteur peut faire le choix d'un site pour l'implantation de l'ouvrage, compte tenu des impératifs non hydrauliques (urbanisation, environnement...).

D'un point de vue hydraulique, l'objectif déterminant est le positionnement du pont dans une section où l'écoulement est au maximum concentré dans le lit mineur et suit une direction peu susceptible de variation dans le temps.

Ce premier choix du site pourra être remis en cause par les phases ultérieures d'élaboration de l'avant-projet.

\section{e) Le choix du débouché de l'ouvrage principal et des ouvrages secondaires}

Dans certains cas, des contraintes imposées par le site, ou des impératifs extérieurs, primeront sur les nécessités hydrauliques, par exemple :

- dans le cas d'une rivière enfermée dans une gorge, la largeur entre culées est imposée par la topographie et l'hydraulique n'est pas un élément de décision ;

- dans le cas d'une voie navigable, le gabarit de navigation impose généralement un tirant d'air, et même parfois une largeur, surabondants pour l'écoulement des crues.

Si le cours d'eau comporte un lit majeur capacitif, c'est-à-dire où les vitesses d'écoulement ne sont pas notables, il est possible de prévoir seulement un pont sur le lit mineur, le lit majeur étant franchi par un remblai formant barrage à l'écoulement. Seuls quelques petits ouvrages (buses par exemple) sont nécessaires sous le remblai, de place en place, pour assurer le remplissage et la vidange du champ d'inondation.

Si le lit majeur est actif, c'est-à-dire si son débit n'est pas négligeable vis à vis de celui du lit mineur, deux types d'ouvrages sont à dimensionner :

- l'ouvrage principal sur le lit mineur dont le débouché est sensiblement de même largeur que le lit mineur;

- les ouvrages dits secondaires, sur le lit majeur, dont il convient de déterminer le nombre, la position et les dimensions de façon telle qu'ils assurent correctement le passage du débit "majeur".

\section{f) Le choix des appuis}

Leur nombre et leurs dimensions sont le résultat des calculs de génie civil. L'hydraulique peut, par contre, imposer des modifications de forme ou les orienter de façon telle que l'obstruction soit la moins brutale possible :

- arrondir les becs des piles;

- orienter les piles dans le sens du courant ou préconiser des piles circulaires si la direction de l'écoulement change en fonction du régime de la rivière.

Un premier avant-projet de structures étant ainsi défini, il importe de déterminer de façon aussi précise que possible les conséquences sur le plan hydraulique des choix qui ont été faits au cours des étapes précédentes. Ces conséquences sont de deux ordres : le remous et les affouillements.

\section{g) Le calcul du remous et des interactions écoulement- structure}

Il s'agit du critère hydraulique qui conditionne le débouché. 
Dans tous les cas, que le lit soit affouillable ou non, il convient d'effectuer le calcul du remous, c'est-à-dire de l'exhaussement du plan d'eau en amont des ouvrages. Les résultats sont à confronter aux impératifs d'environnement : dans le cas d'un ouvrage en zone urbaine des contraintes très sévères peuvent être imposées, par contre dans des régions non urbanisées on peut tolérer une surélévation d'un ou de plusieurs décimètres. Le remous variant en raison inverse de l'ouverture du pont, il constitue donc le critère hydraulique intervenant dans le choix du débouché.

Le problème du remous est l'objet du chapitre II.

\section{h) La détermination des affouillements}

Il s'agit du critère hydraulique intervenant dans le dimensionnement des fondations. Quand le lit est affouillable, ce point est fondamental et conditionne la fixation des cotes de fondation des appuis. Les méthodes qui sont exposées au chapitre III permettent d'obtenir les valeurs prévisionnelles des affouillements dus à la présence des culées et des piles, affouillements dits locaux qu'il convient d'ajouter à une éventuelle évolution générale du profil en long du cours d'eau.

\section{i) L'évolution du profil en long du cours d'eau et la fixation du niveau de référence du lit}

Le profil en long du cours d'eau peut évoluer dans l'avenir, soit que la rivière n'ait pas encore atteint un profil d'équilibre, soit que des aménagements remettent en cause l'équilibre antérieur (calibrages, endiguements, barrages, dragages ....).

Ce point capital sera étudié au chapitre III consacré aux érosions.

\section{j) Le choix des fondations}

S'appuyant sur les résultats de l'étude des sols et des prévisions d'affouillements, le projecteur fixera le type des fondations et leurs dimensions.

De ce choix, dépend en grande partie la stabilité de l'ouvrage dans le cas d'un lit affouillable.

\section{Remous et interactions écoulement-structure}

\section{II.1. Remous}

Les culées de pont provoquent en période de hautes eaux, de par le pincement de l'écoulement, une obstruction qui engendre une variation du niveau de l'eau, sensible parfois, loin en amont ; c'est le phénomène du remous qu'il convient d'étudier dans tous les cas de projets.

Les ponts modernes sont caractérisés par la légèreté de leur structure, en particulier les piles sont minces et ne créent pas d'obstruction importante. Leur effet sur le remous est du second ordre par rapport à celui des culées.

Pour la détermination du remous, nous distinguerons deux cas,

- cas du lit simple ;

- cas du lit composé ; selon que le cours d'eau a pas ou n'a pas de champ d'inondation.

Le remous en lit simple est l'influence sur la surface libre d'une obstruction dans le lit mineur lui-même. Ce problème se limite donc exclusivement au cas des culées faisant saillie dans le lit mineur, le remous n'étant sensible que lorsque l'obstruction est de plus de $10 \%$ de la section offerte à l'écoulement.

Ce cas d'application est donc peu fréquent dans la pratique, louverture d'un pont étant généralement du même ordre de grandeur que la largeur du lit mineur.

Les remous notables se rencontrent dans les cas de lit majeur actif. Comme nous le verrons plus loin, le problème du remous en lit composé est complexe et n'a pas reçu de solution propre; les résultats disponibles ne sont que des adaptations de ceux obtenus en lit simple. C'est pourquoi une aussi grande place sera réservée au cas "académique" du lit simple.

\section{II.1.1. Remous en lit simple}

\section{II.1.1.1. Description du phénomène}

Considérons un lit rectangulaire où l'écoulement est uniforme avant mise en place de l'obstruction.

Sur la figure 3 , représentant une vue en plan de l'écoulement perturbé, on remarque :

- que dans la partie centrale de l'écoulement, la direction des lignes de courant est peu altérée, alors qu'à proximité des rives, elles se "đécollent" des bords aux points : " $a$ ", appelés points de séparation, pour converger vers la contraction, laissant ainsi des zones " $I_{a}$ " dites de "de séparation" qui sont le siège de grandes turbulences. Après la section 2, où elles pénètrent dans la contraction, elles continuent à converger légèrement en se décollant de la culée, jusqu'à la section 3 , à partir de laquelle elles divergent pour occuper de nouveau, à la section 4 (assez éloignée de la contraction), toute la largeur de la rivière. De nouvelles zones de séparation " $I_{a}$ " sont ainsi créées.

Sur la coupe longitudinale (Fig. 2) faite dans l'axe de l'écoulement, on voit que la perturbation apportée par la contraction commence en amont en une section 0 , à partir de laquelle l'eau monte (par rapport à la hauteur normale) jusqu'à un maximum (qui mesure l'importance du remous). Ce maximum est atteint à la section 1 , légèrement en amont de la contraction, au niveau du point de séparation : " $a$ ". La surface libre de l'écoulement commence alors à redescendre pour atteindre son point le plus bas à la section 3 (en-dessous de la hauteur normale) où l'on observe également le minimum de la largeur du jet que constituent les lignes de courant. Elle remonte de nouveau jusqu'à retrouver la hauteur normale. C'est dans la section 3 que les vitesses passent par un maximum.

Dans le rétrécissement, l'écoulement est le plus souvent de régime fluvial; il engendre un écoulement graduellement varié, loin en amont et en aval, bien que dans la contraction elle-même, il soit rapidement varié.

Bien qu'il soit souhaitable, dans certains cas de pouvoir prédire le profil en long complet de la ligne d'eau au droit du pont et loin en amont, la difficulté de décrire cette surface libre tridimensionnelle contraint le projeteur à se contenter de la connaissance de la 


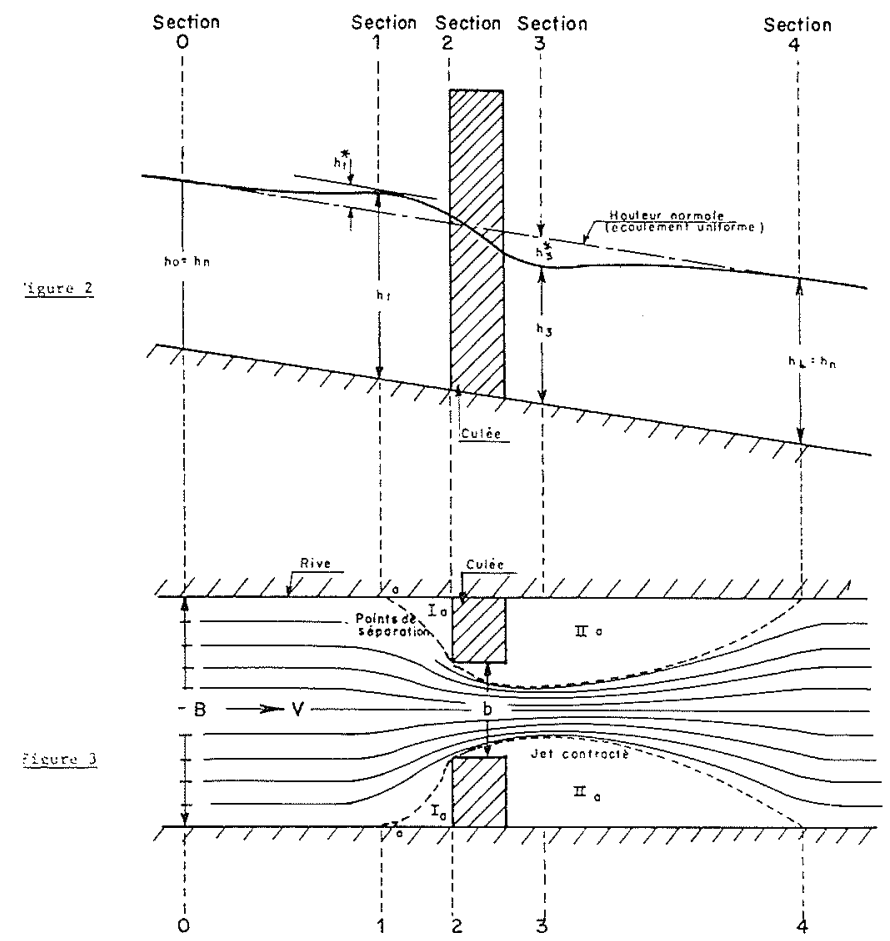

Figure 2 - Remous dû à l'obstruction. Profil en long.

Figure 3 - Remous dû à l'obstruction. Vue du dessus.

caractéristique la plus importante : l'exhaussement maximum de la ligne d'eau en amont, $h *=h_{1}-h_{0}$ sur la figure 2.

C'est à cette grandeur que sont essentiellement consacrées les études sur le remous.

\section{II.1.1.2. Valeur de l'exhaussement maximal amont}

\section{a) Moyens d'étude}

Notons tout d'abord le très grand nombre de paramètres régissant le phénomène. Outre ceux caractérisant le fluide et son écoulement, ceux définissant la contraction sont (Fig. 2 et 3 ) :

- le rapport $b / B$ de la largeur de la section rétrécie à la largeur du cours d'eau appelé généralement rapport de contraction $(M=b / B)$;

- l'angle de l'obstruction par rapport à l'axe de l'écoulement ;

- l'excentricité de l'obstruction

- et enfin, les nombreux paramètres permettant de caractériser la géométrie de l'obstacle : largeur des culées, angle d'ouverture, pente transversale, état de surface.

Deux voies ont été utilisées pour étudier ce problème complexe : la voie analytique et la voie expérimentale.

La méconnaissance du processus des échanges d'énergie, au niveau de la contraction, rend très difficile toute approche théorique, pour laquelle de nombreuses hypothèses sont nécessaires.

De nombreuses études expérimentales, sur modèle réduit, ont conduit, par utilisation de l'analyse dimensionnelle, à l'élaboration de formules empiriques plus simples de maniement pour le projecteur et qui semblent donner une assez bonne approximation [5].

\section{b) Résultats pratiques}

La méthode la plus facilement utilisable est celle du Bureau of Public Roads des USA [4], élaborée à partir d'essais sur modèles et dont la validité a été suffisamment vérifiée pour qu'il soit possible de lui accorder pleine confiance.

Elle est valable, aussi bien dans le cas de l'écoulement uniforme que dans celui de l'existence d'un remous dans la section où est projetée l'implantation de l'ouvrage d'art.

Soient (Fig. 2 et 3) :

$b=$ la largeur entre culées;

$h_{0}=$ le tirant d'eau dans la section considérée avec rétrécissement;

$M=b / B=$ rapport de contraction dans le cas de sections rectangulaires.

Plus généralement, lorsque les sections sont quelconques, $M$ est défini comme le rapport du débit passant dans l'état naturel sous le tirant d'eau $h_{0}$ par la surface qui sera prise comme débouché du point, au débit total de la rivière.

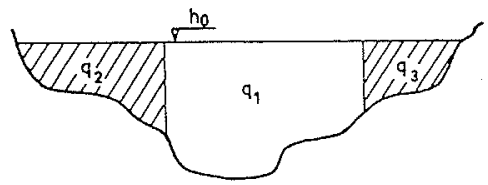

Figure 4 - Calcul de l'exhaussement maximal amont.

dans le cas particulier de la figure 4

$$
M=\frac{q_{1}}{q_{1}+q_{2}+q_{3}}
$$

(Cette définition présente l'avantage de tenir compte de vitesses d'écoulement différentes dans les diverses zones, ce qui est précieux pour l'utilisation de la méthode en lit composé présentée au paragraphe suivant).

$h_{1}=$ le tirant d'eau maximum en amont du rétrécissement

$h_{1}^{*} \square h_{1}-h_{0}=1 \mathrm{e}$ remous maximum dû au rétrécissement;

$V_{a}=Q /\left(b h_{0}\right) \neq$ la vitesse moyenne dans la section rétrécie sous un tirant d'eau égal à celui de l'écoulement non perturbé.

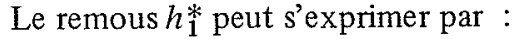

$$
h_{i}^{*}=K^{*} \frac{V a^{2}}{2 g}
$$

en désignant par $K^{*}$ un coefficient qui dépend en premier lieu du rapport de contraction $M$ et de la forme des culées, mais aussi dans une moindre mesure des piles, de l'excentricité et de l'inclinaison du pont par rapport à l'axe du cours d'eau.

Les études ont montré que la largeur des culées était sans influence sur la valeur de $K^{*}$ (essais avec des ponts routiers de 1 à 6 voies de large).

Le coefficient $K^{*}$ peut être facilement calculé au moyen d'abaques selon la décomposition suivante :

$$
K^{*}=K_{b}+\Delta K_{p}+\Delta K_{e}
$$


$K_{b}=$ c'est le coefficient de base, terme principal de calcul. Il est donné par les figures 5 et 6 en fonction du coefficient d'obstruction $M$ pour les divers types de culées représentés en vue de dessus,

$\Delta K_{p}=$ terme correcteur rendant compte de l'effet d'obstruction dû aux piles, fonction de $J$ coefficient d'obstruction des piles (défini sur la figure 7), du type de pile et de $M . \Delta K_{p}$ peut être considéré comme indépendant du diamètre, de la largeur, de l'espacement des piles et du nombre de piles mises dans l'alignement les unes des autres à condition qu'il soit au plus égal à cinq.

L'utilisation de l'abaque de la figure 7 est la suivante : - le graphique $A$ donne pour divers types de piles un coefficient $\Delta K$ correspondant à $M=1$,

- le graphique $B$ donne la correction $o$ en fonction du coefficient d'obstruction $M$.

Le coefficient correcteur dû aux piles est en définitive :

$$
\Delta K_{p}=\sigma \cdot \Delta K .
$$

$\Delta K_{e}=$ coefficient correcteur dû à l'excentricité défini sur la figure 8 en fonction de $M$ et du coefficient d'excentricité $e$.

Dans le cas particulier d'un pont biais une procédure particulière est prévue qui peut être consultée dans [4].

\section{II.1.1.3. Distance d'atténuation du remous}

Il est possible d'évaluer, avec une bonne approximation, la distance d'atténuation du remous d'exhaussement en lit simple, sans avoir recours à un calcul complet de courbe de remous, grâce à une méthode simplifiée [10].

Si le remous $h_{1}^{*}$ est petit devant le tirant d'eau $h_{1}$ (voir Fig. 2), le remous résiduel $h_{1_{r}}^{*}$ (Fig. 9) à une
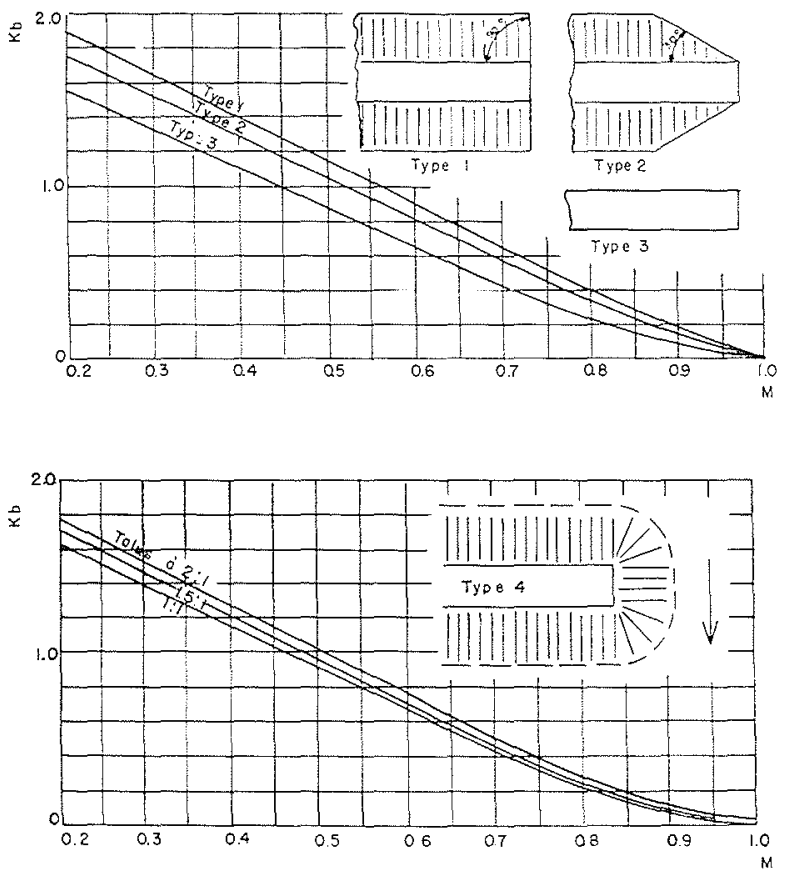

Figures 5 et 6 - Remous en lit simple. Coefficient de base $K_{b}$.
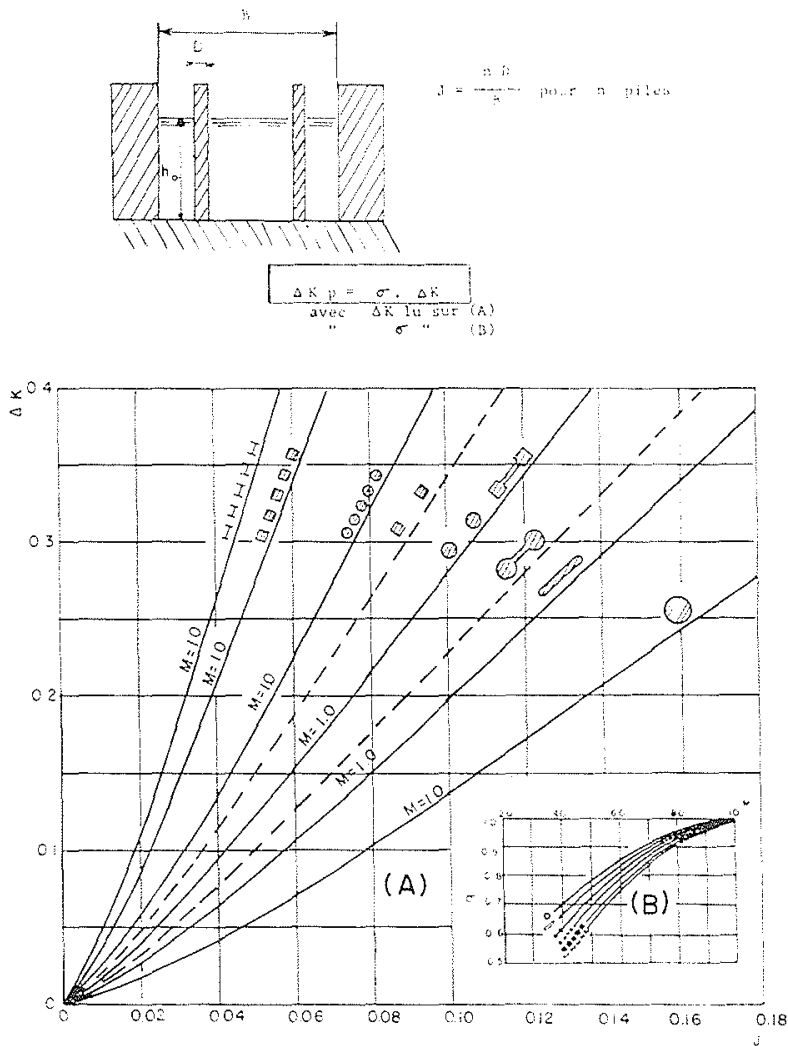

Figure 7 - Remous en lit simple. Coefficient correcteur dû aux piles.
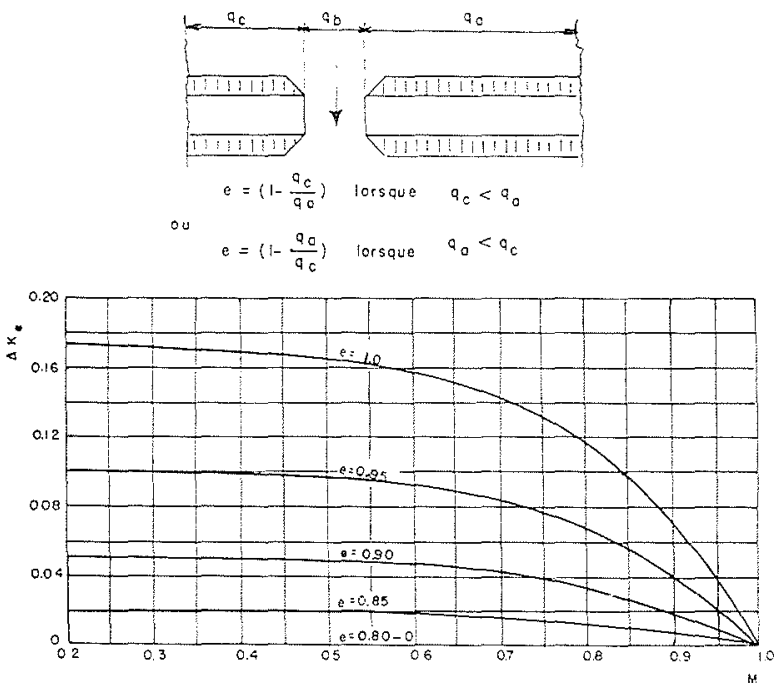

Figure 8-Remous en lit simple. Coefficient correcteur dû à l'excentricité.

distance $x$ en amont peut être approché par l'exponentielle :

avec

$$
h_{1_{r}}^{*}=h_{1}^{*} \cdot e^{-x / x_{0}}
$$

$$
x_{\boldsymbol{a}}=0,3 \frac{h_{1}}{I}\left(1-F_{0}^{2}\right)
$$

$I=$ pente du lit

$F_{0}=$ nombre de Froude de l'écoulement. 


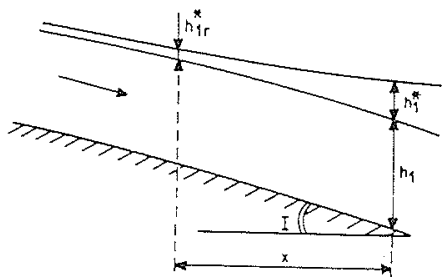

Figure 9 - Calcul de la distance d'attenuation du remous.

Dans la pratique, on peut retenir que :

- à la distance $x_{0}$ la surélévation $h_{1_{r}}^{*}$ est égale à $0,37 h_{1}^{*}$; - la surélévation est réduite au dixième de sa valeur initiale à la distance $2,3 x_{0}$, et au centième à la distance $4,6 x_{0}$.

\section{II.1.2. Remous en lit composé}

\section{II.I.2.1. Difficulté du problème}

Dans le cas très fréquent où la voie à créer devra franchir un cours d'eau et son champ d'inondation, il convient de déterminer le remous dû à l'ouvrage principal sur le lit mineur et à ses voies d'accès, éventuellement percées d'ouvrages secondaires, sur le lit majeur.

Le problème est infiniment plus complexe que dans le cas du lit simple, en raison de l'inégalité des vitesses de l'écoulement et des échanges de débit possibles entre lit mineur et lit majeur. Le grand nombre de paramètres en jeu explique aisément qu'aucun code de calcul ne permette, dans le cas général, de prévoir l'exhaussement maximal de la ligne d'eau.

Le recours au modèle réduit peut parfois constituer une approche : cependant pour obtenir une répartition correcte du débit en amont de l'obstruction, il est nécessaire que le modèle soit suffisamment long; pour représenter correctement l'écoulement au travers des pertuis, il faut une échelle suffisamment grande. Ceci explique que cette technique soit coûteuse et aussi délicate de mise en oeuvre.

La méthode la plus couramment utilisée consiste à évaluer par le calcul la valeur du remous.

\section{II.1.2.2. Méthodes pratiques d'évaluation de l'exhaus- sement maximal}

Deux cas distincts sont à envisager :

\section{a) Cas du lit majeur purement capacitif}

Il en est ainsi lorsque l'étude hydraulique de l'état naturel a permis de conclure que l'écoulement dans le lit majeur n'est pas notable : très faible submersion ou obstacles importants à l'écoulement créant une très forte rugosité.

Dans de tels cas, le lit majeur est simplement à négliger dans un calcul de remous, il convient d'utiliser la méthode en lit simple en supposant que l'intégralité du débit de crue est concentrée dans le lit mineur.

\section{b) Cas du lit majeur actif}

Les vitesses d'écoulement dans le lit majeur ne sont pas négligeables.

Le problème est alors complexe et peut recevoir l'une des quatre solutions ci-dessous:

$\alpha$ ) disposer des ouvrages de décharge dans le lit majeur, ceux-ci étant calculés de telle sorte que la répartition de débit entre lit mineur et lit majeur soit conservée;
B) diminuer le débit dans le lit majeur en concentrant une plus grande partie du flot dans le lit mineur;

$\gamma)$ supprimer totalement l'écoulement dans le lit majeur par l'absence d'ouvrage de décharge ;

o) à l'inverse des deux cas précédents, augmenter le débit du lit majeur au détriment du lit mineur.

Une méthode employée pour le calcul du remous consiste à traiter indépendamment l'un de l'autre, le lit mineur et le lit majeur, en les supposant sans communication, l'objectif étant d'obtenir le même remous dans ces deux lits pour éviter des écoulements transversaux :

- calcul de l'exhaussement dû à l'ouvrage sur le lit mineur pour le débit mineur adopté ; on fera appel dans ce cas à la méthode exposée pour les calculs de remous en lit simple :

- calcul du débouché des ouvrages secondaires permettant d'écouler, sous le même exhaussement que dans le lit mineur, le débit du lit majeur ; ces ouvrages peuvent être assimilés soit à $n$ lits mineurs et calculés comme tels, soit à des buses selon le cas.

A remous donné, le choix entre les quatre types de solution ci-dessus est de nature économique, il revient à apprécier le coût d'ouvrages de décharge en lit majeur vis à vis du coût d'augmentation du débouché de l'ouvrage principal et de création éventuelle d'endiguements ou de murs guideaux (voir § III.2.2.4.c.).

Il convient aussi de s'assurer que la solution choisie ne conduise pas à des conditions d'écoulement risquant de remettre en cause la stabilité du lit.

\section{II.1.3. Conclusion sur les remous}

L'implantation d'un pont, et en particulier de ses culées, crée en période de hautes eaux une déformation de la surface libre du cours d'eau. Ce phénomène tridimensionnel est assez mal connu.

Le projecteur de pont désire avant tout connaitre l'exhaussement maximal de la ligne d'eau de crue en amont du rétrécissement.

Dans le cas d'une rivière concentrée dans son lit mineur, une méthode empirique d'application aisée permet de calculer cette grandeur avec une précision suffisante.

Pour un écoulement avec plaine d'inondation, le problème est plus complexe. Il peut seulement être fait appel à des extensions de la méthode en lit simple qui aboutissent à un choix économique.

\section{II.2. Intéractions écoulement-structure}

Les phénomènes dont il est question ici sont dus à la présence des piles de pont dans l'écoulement. Ils sont de deux ordres:

- action des structures sur le fluide : instabilités de l'écoulement au droit de l'ouvrage;

- action du fluide sur les structures : efforts hydrodynamiques sur les piles. 


\section{II.2.1. Oscillations du plan d'eau}

\section{II.2.1.1. Description du phénomène}

Sous certaines conditions, l'écoulement entre des piles de pont peut être instable par suite d'oscillations de la surface libre entre les piles. Ce phénomène est dû au lacher des tourbillons alternés de Bénard-Karman dans le sillage des piles. Marvaud et Ramette [7] ont étudié expérimentalement, puis analytiquement ces oscillations; ils ont mis en évidence deux modes distincts d'oscillation:

- un mode longitudinal : à l'intérieur des passes, des ondes cylindriques perpendiculaires aux parois et en opposition de phase progressent de l'aval vers l'amont (Fig. $10 \mathrm{a}$ et b).

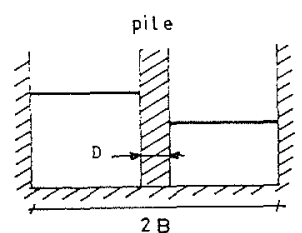

a) Vue de face

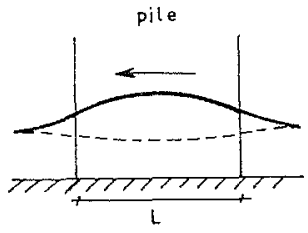

b) Vue de profil

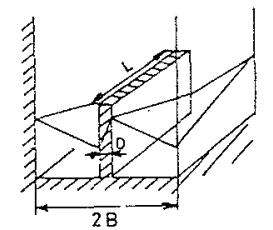

Figure 10 - Oscillations du plan d'eau dans le sillage des piles.

- un mode transversal : apparaissant pour des largeurs de passe relativement grandes par rapport à la longueur de la pile; l'onde n'est plus cylindrique, il existe un nœud au milieu de chaque passe (fig. 10c).

Les conditions des essais sont :

- des nombres de Reynolds de l'écoulement

$$
10^{3}<R<10^{5},
$$

- des nombres de Froude $0,10<$ II $<0,40$

- et des piles telles que $L / D>2$.

L'étude a porté principalement sur les oscillations longitudinales et a montré qu'elles n'apparaissent que dans une gamme déterminée de nombre de Froude

$$
\text { भI }=V / \sqrt{g H}
$$

et du nombre de Strouhal

$$
\mathfrak{S}=D / V T
$$

en désignant par $V$ la vitesse moyenne de l'écoulement,

$H$ le tirant d'eau

$T$ la période des oscillations.

En particulier le début des oscillations se produit pour $\mathscr{S} \simeq 0,32$, leur maximum pour $\mathscr{S} \simeq 0,22$ et leur fin pour $\mathfrak{S} \simeq 0,20$.

\section{II.2.1.2. Prévision des oscillations}

Soient $n$ piles de longueur $L$ et de largeur $D$ régulièrement espacées dans un canal de largeur $2 B$. Posons $l=4 B / n$
Il a été montré qu'une relation fonctionnelle existe entre $M=\frac{T \sqrt{g H}}{l}$ et $\frac{L}{l} \quad$ (voir fig. 11)

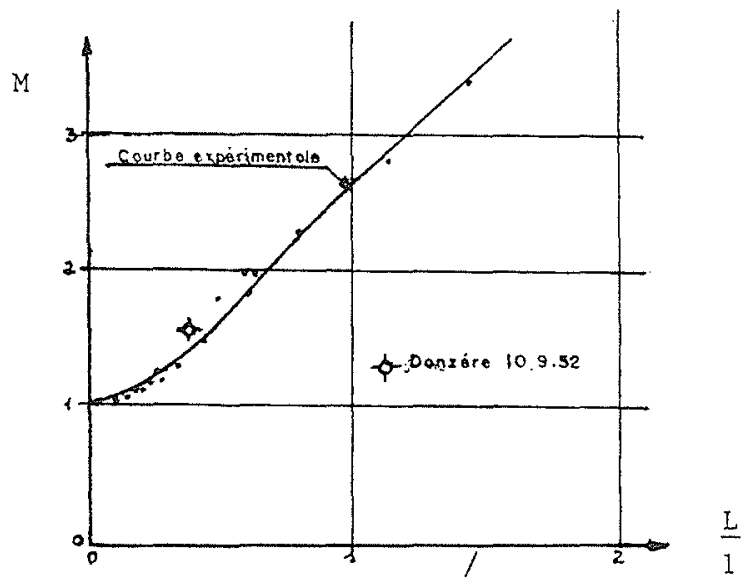

Figure 11 - Loi des oscillations entre les passes.

Connaissant $L / l$, l'ordonnée correspondante de la courbe ci-dessus donne une valeur de $M$. En portant dans $\mathscr{J}=D / M l \mathfrak{S}$, les valeurs 0,32 et $0,20 \mathrm{de} \mathfrak{S}$, on obtient le domaine de $\mathscr{J}$ d'apparition des oscillations longitudinales.

En nature où les nombres de Reynolds $\mathcal{R}$ sont de l'ordre de $10^{6}$, les oscillations doivent apparaitre pour des valeurs de ฐ̆ légèrement inférieures à celles déterminées par la courbe figure 11 (voir le point nature du barrage de Donzère).

Gallez [6] propose une méthode moins approchée mais plus complexe d'utilisation à laquelle on pourra faire appel dans le cas délicats.

\section{II.2.2.3. Conclusion}

$\mathrm{Si}$ les caractéristiques d'un avant-projet laissent prévoir la possibilité d'instabilités de la surface libre dans la gamme des conditions hydrauliques à envisager, deux remèdes sont possibles :

- soit modifier la période propre de l'oscillateur : changer la longueur des piles;

- soit modifier la fréquence des tourbillons alternés en jouant sur la forme de l'extrémité aval des piles (bord de fuite en dièdre).

Notons enfin que ces phénomènes d'oscillations très spectaculaires et dangereux dans le cas de piles assez rapprochées (exemple du barrage de Donzère sur le Rhône), sont d'amplitude plus modeste pour les ponts. Elles peuvent néanmoins être une gêne pour la navigation fluviale et exacerbent de toutes façons les affouillements au pied des piles.

\section{II.2.2. Portance et trainée des piles}

\section{II.2.2.1. Description du phénomène}

Les piles de pont peuvent subir, sous l'effet de l'écoulement, des forces de portance et de traînée qui sont importantes même pour des inclinaisons de l'écoulement de quelques degrés par rapport à l'axe de la pile.

Quand les oscillations décrites au paragraphe précédent prennent naissance les forces deviennent très fluctuantes et par conséquent dangereuses. 


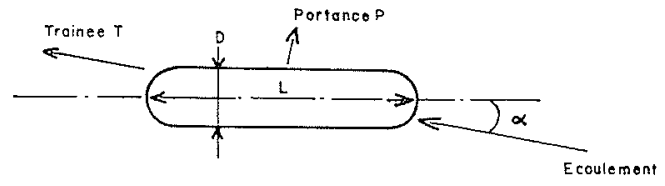

Figure 12 - Coupe d'une pile allongée inclinée sur le courant.

Ces phénomènes ont été étudiés expérimentalement par Apelt et Isaacs [8]. Les essais effectués pour des nombres de Reynolds $\mathcal{Q} \simeq 10^{4}$ ont conduit à la détermination de coefficients de traînée $C_{D}$ et de portance $C_{L}$; qu'il serait hasardeux de vouloir transposer par les Reynolds nature; ils permettent cependant de comparer le comportement de trois types de piles représentées par la figure 13 .

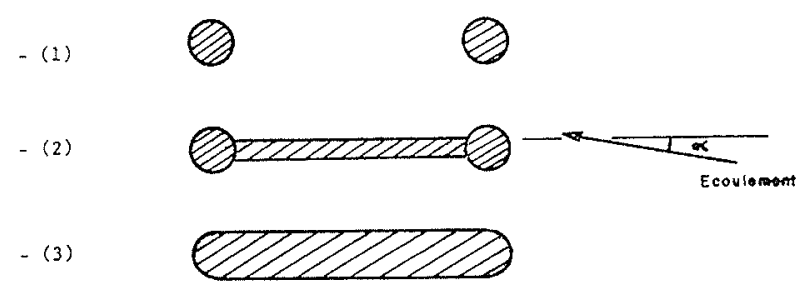

Figure 13 - Types de piles étudiées pour les efforts dûs à l'écoulement.

Pour les types (2) et (3) un angle d'incidence $\alpha$ de quelques degrés seulement donne des valeurs de $C_{D}$ et surtout de $C_{L}$ assez importantes. Par contre, pour les piles jumelles du type (1) ces coefficients restent modérés et très peu sensibles à la variation de $\alpha$.

\section{II.2.2.2. Conclusion pratique}

Indépendamment des questions d'affouillement, les problèmes d'efforts sur les piles doivent faire adopter avec prudence toute forme allongée dès que l'angle d'incidence $\alpha$ du courant par rapport à l'axe de la pile peut ne pas être nul pour certaines des conditions hydrauliques possibles. Dans ce cas, une succession de fûts circulaires est la seule forme à conseiller qui soit insensible à l'influence de l'angle d'incidence.

\section{Erosions}

Dans le cas où le lit est affouillable le problème des érosions est capital, de lui dépend la pérennité de l'ouvrage.

Les études entrant dans la rubrique "érosions" ont été énumérées au chapitre présentant l'articulation de l'élaboration d'un projet ( $\$$ I.2). Ce sont :

- l'étude morphologique du cours d'eau se subdivisant en :

- étude de la stabilité en plan,

- étude de l'évolution du profil en long en vue de définir un niveau de référence du lit.

- la détermination des affouillements locaux dûs aux piles et aux culées qui sont à ajouter à l'évolution générale du lit.
Ces divers points vont être envisagés successivement ainsi que les moyens propres à prévenir les érosions.

\section{III.1. Evolution générale du lit à long terme en vue de définir un niveau de référence}

\section{III.1.1. Rivières stables en plan}

\section{III.1.1.1. Problème de l'évolution du profil en long}

Les berges du lit mineur sont fixées; il y a pratiquement coïncidence du lit mineur et du lit moyen suivant un tracé plus ou moins sinueux.

$\mathrm{Si}$ les renseignements disponibles, sur une assez longue période, permettent de pressentir que le cours d'eau n'a pas atteint son profil d'équilibre, il convient de procéder à une étude pour prévoir quel sera ce niveau final d'évolution des fonds.

L'étude doit porter sur un très long bief de façon telle que les conditions aux limites puissent être parfaitement fixées. Ceci exclut donc le modèle physique dont les dimensions et les difficultés d'exploitation seraient prohibitives.

Les seuls procédés permettant de répondre à la question sont, soit les modèles mathématiques d'érosion, soit les comparaisons des cotes moyennes des profils en travers du lit levés à quelques années d'intervalle et qui permettent d'ailleurs l'étalonnage des modèles mathématiques, ou alors l'application de règles empiriques de morphologie fluviale.

III.1.1.2. Modèle mathématique d'érosion, ses limites

Il s'agit de modèle filaires monodimensionnels. En d'autres termes, chaque section en travers est décrite par une cote du plan d'eau $\eta$, une seule cote $Z_{f}$ du fond moyen et une seule vitesse moyenne $U$ dans la section (Fig. 14).

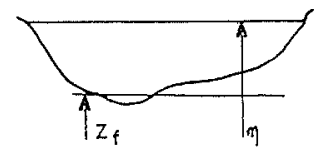

Figure 14 - Modèle filaire monodimentionnel.

Le transport des matériaux par charriage est décrit par une loi physique de début de charriage et une fonction "débit solide" du type Meyer-Peter ou Einstein. Cette loi n'étant valable que pour un charriage uniforme, on doit la compléter par des lois de charriage en régime graduellement varié (loi de chargement s'il existe un gradient longitudinal positif de débit solide).

La multiplicité des coefficients empiriques qu'introduisent ces lois nécessite de disposer d'un bon "historique" de l'évolution des fơnds pour étalonner correctement ces modèles.

Par ailleurs ces modèles ne peuvent prédire qu'une évolution des fonds moyens et on comprend aisément que cette information soit d'autant plus proche de la réalité que le charriage s'effectue dans le fleuve réel sur lit plat ou sans inégalité transversale. C'est le cas pour les fleuves à matériaux assez grossiers (Rhône, Rhin) et dans les biefs sans grande courbure du chenal où s'effectue le charriage.

Dans le cas contraire (Loire, chenal courbe) il faut ajouter aux valeurs trouvées les érosions locales dues aux 
courants secondaires. L'emploi de ces modèles est alors très délicat.

On trouvera des exemples dans les ouvrages suivants cités en références [12], [26], et [27].

\section{III.1.2. Rivières instables en plan}

\section{III.1.2.1. Le phénomène des méandres}

Dans des cours d'eau dont le lit moyen est peu sinueux et le débit suffisamment important, les berges sont trop éloignées pour avoir un effet directeur sur les débits d'étiage. Le chenal des basses eaux divague entre les berges.

Les méandres engendrent des courants hélicoïdaux qui érodent la rive convexe et créent des atterrissements dans la concavité de la courbe. Cette évolution du profil en travers augmente l'amplitude des méandres et les fait progresser vers l'aval.

Des études ont mis en évidence la constance de certains ratios des caractéristiques géométriques des méandres [11].

L'ingénieur s'intéresse surtout à la fixation des berges pour éviter des divagations du lit particulièrement préjudiciables à l'implantation de tout ouvrage.

\section{III.1.2.2. Correction des rivières}

Une action locale peut apporter une stabilité temporaire du lit, mais à la faveur de circonstances exceptionnelles la rivière réussira presque inéluctablement à contoumer les dispositifs (voir l'exemple du Logone au Tchad en annexe).

Pour obtenir une stabilité durable, une action de grande envergure est nécessaire sur tout un bief. II n'est qu'à considérer les travaux de Fargue sur la Garonne, de Girardon sur le Rhône ou de Tulla sur le Rhin.

Les méthodes de correction s'appuient sur divers procédés [13] allant de l'utilisation des digues, épis, tenons, aux dragages ou à l'utilisation de panneaux de fond. Chaque cours d'eau constitue alors un cas spécifique.

\section{III.1.3. Influence des aménagements sur la stabilité du lit}

Lors de l'étude d'un projet dans une section donnée d'un cours d'eau, il ne suffit pas de s'assurer de la stabilité du lit depuis une longue période, il convient aussi de vérifier qu'aucun aménagement n'est prévu, aménagement risquant de modifier le transport solide et par conséquent de mettre en cause l'équilibre du lit :

- barrage en amont arrêtant le transport solide et obligeant le charriage à s'alimenter sur les matériaux en place, d'où un creusement du lit en aval du barrage [12].

- dragage, recalibrage ou extraction de matériaux en aval entraînant un abaissement des lignes d'eau, d'où une érosion régressive remontant jusqu'au droit de l'ouvrage (voir l'exemple de l'Agly en annexe).

Si un aménagement de ce type est construit sans précaution particulière et que les fondations du pont soient mises en péril par l'abaissement du niveau du lit, une méthode capable d'enrayer le processus consiste à édifier un seuil à l'aval immédiat des ouvrages ou un radier assurant sous le pont une stabilisation des fonds à une cote donnée.

\section{III.1.4. Conclusion}

La prévision de l'évolution à long terme du lit d'une rivière est un domaine particulièrement délicat. Autant il est simple de connaitre qualitativement le sens de l'évolution, autant il est difficile de fournir des prévisions quantitatives.

Une voie semble s'ouvrir actuellement avec la mise au point de modèles mathématiques, mais ces outils sont encore trop délicats d'exploitation pour pouvoir être utilisés par des non spécialistes. Leurs résultats sont à analyser avec prudence; ils nécessitent pour être étalonnés, une connaissance précise de l'évolution moyenne des fonds sur une période la plus longue possible. Si l'ouvrage à construire entraîne, de par les endiguements ou les culées, un rétrécissement sensible du lit, un ordre de grandeur de l'abaissement du fond, qui se superpose d'ailleurs à l'abaissement général, est donné par la formule des rétrécissement longs citée en (III.2.2.2.b).

\section{III.2. Erosions locales}

Deux éléments de structure peuvent être implantés dans le lit du fleuve et engendrer des affouillements lorsque les matériaux y sont sujets, ce sont les culées d'une part et les piles d'autre part.

Nous envisagerons successivement les méthodes permettant de prédire l'ampleur de ces affouillements et les moyens propres à les éviter.

\section{III.2.2. Affouillements dus aux piles}

De nombreuses études ont été consacrées aux piles circulaires qui ont permis d'élaborer des lois expérimentales de maximum d'affouillement. Nous envisagerons d'abord ce cas idéal dont l'intérêt n'est pas seulement académique puisque de nombreux ouvrages sont bâtis sur ce type de piles. Se reporter au chapitre II.2 consacré aux efforts exercés sur les piles, dans lequel a été souligné le danger des piles allongées sous l'effet d'un courant incliné de quelques degrés seulement par rapport à l'axe de la pile.

\section{III.2.1.1. Affouillement au pied des piles cylindriques circulaires}

\section{a) Description du phénomène}

La couche limite de fond est déformée par la présence de la pile, il se forme un tourbillon en fer à cheval qui présente un maximum d'intensité au niveau de la génératrice amont de la pile. Il induit des vitesses verticales qui agissent comme une tarrière dans le lit, donnant un cône d'affouillement dont le point extrème se situe sur le plan de symétrie à quelque distance en amont du pied de la pile.

La cinématique de cet écoulement tridimensionnel n'a pas pu être caculée. De nombreuses études expérimentales se sont focalisées sur l'affouillement et en particulier sur la profondeur maximale susceptible d'être atteinte.

En 1956, Chabert et Engeldinger [14] ont montré les premiers, à partir des résultats de nombreux essais sur modèle, que pour une pile et un matériau de fond donné, la profondeur maximale atteinte ne pouvait 
dépasser un certain seuil obtenu pour les conditions de début de charriage continu du matériau en l'absence d'obstacle.

Au delà du débit donnant le début de charriage, la profondeur d'affouillement fluctue au gré des dunes.

\section{b) Loi du maximum de l'affouillement [15]}

Une approche par l'analyse dimensionnelle permet de prévoir que la loi donnant l'affouillement maximummaximorum $P_{m}$, pour une pile de diamètre $D$ et un matériau de fond, de granulométrie uniforme $d$, dans un écoulement de tirant d'eau $h$, serait de la forme :

$$
\frac{P_{m}}{D}=f\left(\frac{d}{D}, \frac{h}{D}\right)
$$

sì on ne considère que les sédiments naturels ont une densité voisine de 2,6 .

Pour des milieux granulaires, c'est-à-dire sans aucune cohésion, l'influence du paramètre $d / D$, caractérisant le diamètre du sédiment, n'a pas pu être mise en évidence de façon significative.

Le seul paramètre significatif semble être $h / D$ qui caractérise la profondeur de l'écoulement. La totalité des auteurs s'accordent à constater que ce paramètre $h / D$ n'a plus d'influence au-delà d'une valeur de l'ordre de 2 . Par contre, pour les faibles profondeurs, une relation empirique est nécessaire ; on peut adopter celle proposée dans [15] :

$$
\frac{P_{m}}{D}=1,5 \tanh \left(\frac{h}{D}\right)
$$

Comme le montre la figure 15, une certaine dispersion des points expérimentaux existe, ce qui conduit à conseiller d'adopter dans tous les cas de détermination de la profondeur extrémale $P_{m}$ d'un projet :

$$
P_{m}=2 D
$$

\section{c) Limites d'application de la loi}

La loi du double du diamètre permet de prédire l'affouillement maximal susceptible d'être atteint dans le cas d'un écoulement fluvial et lorsque les matériaux

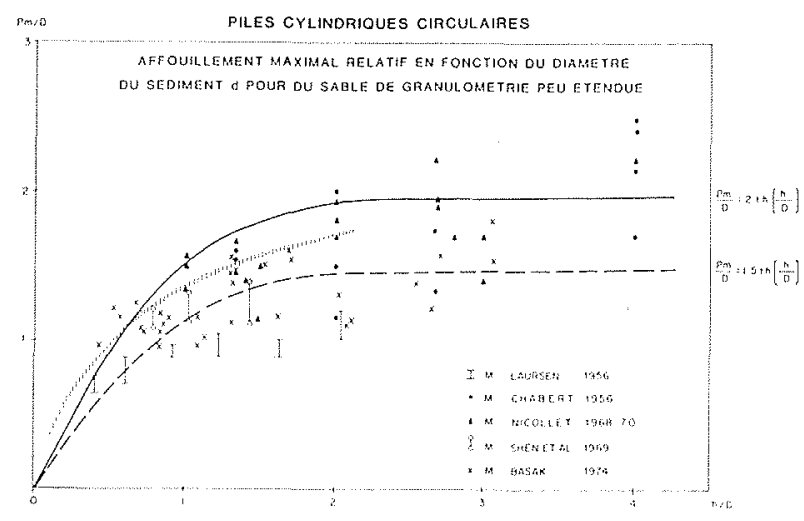

Figure 15 - Affouillement maximal au pied des piles de pont cylindriques circulaires. $\mathrm{du}$ lit sont suffisamment petits ( $d$ quelques $\mathrm{cm}$ ) et non cohésifs. Aucun renseignement valable n'est actuellement disponible en ce qui conceme le torrents.

Pour les rivières naturelles où les sédiments présentent toujours une certaine étendue granulométrique, des essais effectués au Laboratoire National d'hydraulique de Chatou ont montré que les valeurs limites trouvées pour le diamètre moyen constituent le maximum susceptible d'être atteint quelles que soient les conditions d'écoulement [15].

Pour les sols cohésifs, aucune méthode valable ne permet actuellement de prédire l'affouillement, mais il semble raisonnable de supposer que la loi du double du diamètre soit encore valide [16].

Il est important de penser à divers phénomènes spéciaux capables de modifier de façon notable l'affouil lement, ce sont :

- l'amoncellement de corps flottants à l'amont d'une pile, ce qui augmente ses dimensions:

- les dunes et bancs de sables qui peuvent changer l'angle d'attaque et abaisser le niveau de base du lit :

- l'effet des crues très rapides qui peuvent engendrer des affouillements particulièrement importants en raison de conditions non permanentes du transport solide.

\section{III.2.1.2. Cas des piles non circulaires}

Une multitude de formes est possible. Un cas fréquemment rencontré est celui des piles hémicylindriques constituées par un parallélipipède de longueur $L$ dont les deux extrémités sont des demi-cylindres circulaires de diamètre $D$ (fig. 16).

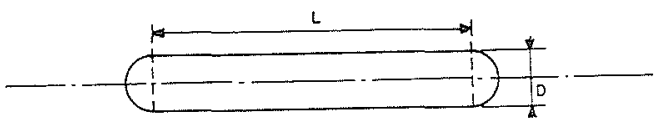

Figure 16 - Coupe d'une pile hémicylindrique.

Des essais en canal [15] ont montré que ce type de pile, disposé avec un angle d'incidence nul dans le courant, donne des affouillements ne dépassant pas sensiblement ceux trouvés pour la pile circulaire de diamètre $D$, c'est-à-dire de même maître-couple. Il est donc possible d'utiliser l'expression $P_{m}=2 D$ dans ce cas.

Pour une pile rectangulaire aux extrémités non arrondies, il semble prudent d'adopter un coefficient multiplicateur de l'ordre de 1,3 pour l'affouillement, d'où $P_{m}=2,6 D$.

Pour une incidence non nulle, les affouillements peuvent atteindre des valeurs considérable (voir en annexe, les exemples du Canada ou du Big Sioux Rivers aux U.S.A.), il ne faut donc utiliser ce type de piles que dans les sites où la direction de l'écoulement est parfaitement axiale à tous les régimes.

On pourra trouver dans [15] des courbes donnant le coefficient multiplicateur à utiliser en cas d'incidence non nulle.

Les fûts circulaires (éventuellement plusieurs alignés) constituent le type de pile recommandé lorsque l'écoulement est oblique. Cette conclusion avait déjà été tirée de l'étude des efforts sur les piles (chapitre II.2.2.2.). 


\section{II.2.1.3. Protection des piles contre les affouillements}

La forme de la pile ayant été choisie au mieux pour minimiser les affouillements, des dispositions supplémentaires peuvent être envisagées pour empêcher la formation de la fosse ce qui permet de réduire la profondeur des fondations. C'est "in fine" le critère coût qui permet de décider de l'opportunité de ces dispositions.

Les principaux dispositifs de protection expérimentés qui ont donné des résultats intéressants sont :

- un caisson autour de la pile arasé sous le niveau moyen des fonds;

- des structures additionnelles comme de petites piles placées en amont de la pile proprement dite;

- et enfin des tapis d'enrochements.

\section{a) Caisson de fondation}

Des raison de génie civil peuvent entraîner l'obligation de fonder la pile sur un caisson de dimensions supérieures.

Dans ce domaine, Chabert et Engeldinger ont étudié une pile circulaire fondée sur un caisson (Fig. 17). Des essais en canal expérimental leur ont permis de conclure que le dispositif optimal leur apparaît constitué par un caisson de diamètre triple de celui de la pile, dont la cote d'arasement sous le terrain naturel est de l'ordre de la moitié du diamètre de la pile. L'affouillement serait, par ce moyen, réduit au tiers seulement de la valeur atteinte avec la pile seule.

Shen et Schneider ont étudié une variante du système du caisson dans laquelle le caisson est entouré d'une plaque verticale débordante (coffrage de palplanches recépées dans la pratique) (Fig. 18).

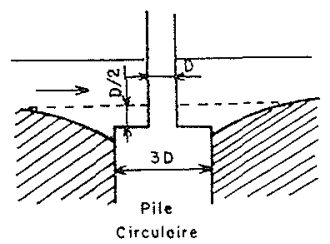

Figure 17 - Coupe dans l'axe d'une pile cylindrique sur caisson de fondation.

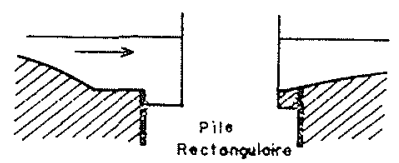

Figure 18 -Coupe dans l'axe d'une pile rectangulaire sur caisson de fondation avec plaque verticale débordante.

L'idée directrice est de contenir le tourbillon en fer à cheval dans une enceinte qui lui permette de s'échapper vers l'aval. Ces essais ont porté sur des piles rectangulaires.

Dans les conditions optimales de dimension de l'ensemble, il a pu être obtenu un niveau des fonds affleurant le rebord en amont et sur les côtés de la pile ainsi qu'un remblaiement en aval. La plate-forme ne semble pas devoir être aussi étendue en aval qu'en amont de la pile.

Ce dispositif a permis de réduire l'afouillement de moitié. Les dimensions de la pate-forme et du rebord par rapport à la pile et aux autres paramètres (écoulement, sédiment) n'ont pas été étudiées suffisamment d'une façon systématique pour permettre de tirer des lois sur les dimensions valables dans le cas général.

Les rebords permettent assurément de diminuer les dimensions du caisson.

b) Structures additionnelles placées en amont : pilots

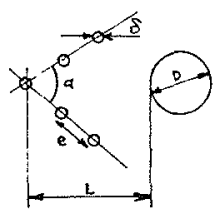

Figure 19 - Vue en plan d'une pile cylindrique et de pilots amont.

Des structures additionnelles sont parfois prévues pour protéger les piles de pont des chocs dus aux corps flottants : exemple des Duc d'Albe sur les voies navigables.

Chabert et Engeldinger [14], puis Chang et Karim (1969) ont étudié l'implantation de petites piles en amont de la pile proprement dite (Fig. 19). L'idée directrice est de briser le courant incident et par làmême d'affaiblir le tourbillon générateur de l'érosion.

Le nombre de paramètres nécessaires à la définition d'une telle structure est très important : $n$ pilots de diamètre $\delta$, distants de $e$, ouverts selon l'angle $\alpha$ et à la distance $L$ de la pile, soit 5 paramètres. Aucune loi générale n'a pu être élaborée concernant un tel dispositif.

Les essais de laboratoire ont permis de constater des réductions d'affouillement atteignant $50 \%$.

\section{c) Tapis d'enrochement}

Il s'agit ici de la méthode la plus couramment utilisée dans la pratique pour remédier à des érosions constatées : on déverse des blocs dans la fosse d'affouillement. De multiples auteurs se sont penchés sur ce problème - voir la liste dans [21] - et ont élaboré des recommandations sur le choix des enrochements. L'expérience prouve que ce type de protection est le seul qui permette d'éviter totalement les affouillements.

Carstens (1966) s'appuie sur le fait que la vitesse maximale, autour d'un cylindre en écoulement bidimensionnel, est approximativement le double de la vitesse de l'écoulement non perturbé, pour avancer que la vitesse d'écoulement donnant le début d'affouillement au pied de pile doit être moitié de celle correspondant au début de charriage généralisé.

Les essais de S. Hancu [23] confirmés par Ramette et Nicollet [15] ont effectivement montré, que pour un sédiment donné, un affouillement commence à apparaître au pied d'une pile circulaire pour une vitesse d'écoulement égale à la moitié de la vitesse critique et ce, quel que soit le diamètre de la pile.

Ce résultat permet de déterminer le poids d'enrochements capable de prévenir toute érosion. En effet, dans une rivière où la vitesse extrème d'écoulement en crue est $V_{\max }$, il suffit de disposer des blocs pour lesquels la vitesse de début de charriage est $v_{c}=2 V_{\max }$.

Cette vitesse extrème $V_{\max }$ peut être, avec une précision suffisante, évaluée par le calcul de la vitesse moyenne $Q / S$ de la crue maximale considérée. 
La détermination du diamètre des blocs $\Delta$ en fonction de la vitesse $V_{c}$ peut être faite par exemple au moyen de la formule d'Isbach [22] représentée graphiquement sur la figure 20 et par l'équation suivante :

$$
V_{c}=1,2 \sqrt{2 g \frac{\bar{\omega}_{s}-\bar{\omega}}{\bar{\omega}} \cdot \Delta}
$$

dans le système $M K S$, en désignant par $\bar{\omega}_{s}$ et $\bar{\omega}$ les poids volumiques des blocs et de l'eau.

Pour des blocs de densité 2,6 , on obtient la formule pratique suivante :

$$
\Delta \simeq V_{\max }^{2} / 10
$$

dans le système $M K S$ avec :

$-\Delta=$ diamètre des enrochements en $m$

$-V_{\max }=$ vitesse maximale du cours d'eau en $\mathrm{m} / \mathrm{s}$.

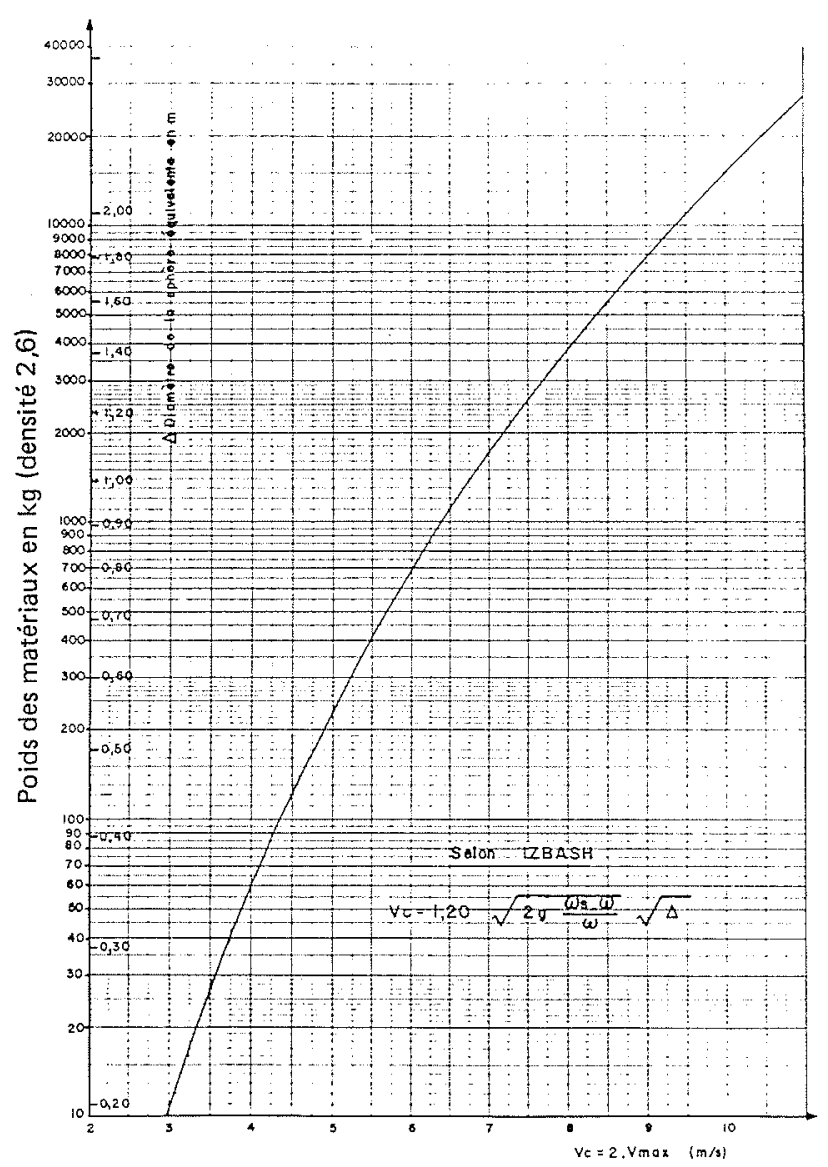

Les dimensions du tapis à envisager pour éviter tout affouillement sont, en plan, de l'ordre de 3 fois le diamètre de la pile pour une pile circulaire, ce qui correspond à l'emprise totale de la fosse d'affouillement. En épaisseur, il est suggéré de prendre la plus grande des deux valeurs suivantes : la dimension de la pile ou le triple du diamètre des enrochements. La constitution d'un bon filtre est nécessaire pour éviter que les blocs ne s'enfoncent dans le lit.

Il est aussi essentiel que les enrochements ne constituent pas au pied de chaque pile un monticule qui crée une obstruction importante à l'écoulement.

\section{d) Conclusion}

Bien que des résultats intéressants puissent être obtenus au moyen du caisson de fondation arasé sous la cote du lit ou au moyen de pilots disposés en amont de la pile, aucune loi générale ne permet actuellement de dimensionner ces ouvrages de protection.

Par contre, une méthode simple et efficace, permettant d'empêcher tout affouillement, consiste à disposer un tapis d'enrochements dont la dimension des blocs peut être évaluée uniquement à partir de la connaissance de la vitesse extrême du cours d'eau.

\section{III.2.2. Affouillements dus aux culées}

L'affouillement dû aux culées a été beaucoup moins étudié que celui dû aux piles. La raison en est vraisemblablement que la plupart des accidents de ponts ont été causés par la ruine des piles.

Le phénomène étant encore plus complexe que pour les piles, on conçoit que les résultats disponibles soient moins élaborés et moins sûrs.

\section{III.2.2.1. Description du phénomène}

Dans un cours d'eau, à lit affouillable, les culées d'un pont peuvent avoir un double effet sur les fonds :

- chaque culée est une sorte d'éperon partant de la rive et s'avançant dans l'écoulement. Comme pour les piles, il se crée une nappe tourbillonnaire qui est responsable de la formation d'une fosse d'affouillement de forme approximativement conique [17]. L'angle des génératrices du cône avec la verticale est compris entre $20^{\circ}$ et $30^{\circ}$ (Fig. 21).

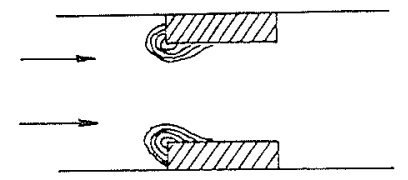

Figure 21 - Affouillement local au pied d'une culée.

Cet affouillement est dit "affouillement local", c'est celui que l'on peut observer au voisinage d'une culée saillant au bord d'un écoulement de largeur infiniment grande.

- l'ensemble des deux culées provoque un rétrécissement du lit, d'où une augmentation des vitesses d'écoulement et une érosion dans la section rétrécie. L'érosion due au rétrécissement s'ajoute à l'affouillement local dû à chaque culée et, dans de nombreux cas d'observation nature, il est bien difficile de séparer ces deux composantes de l'affouillement.

Les études consacrées à ces deux types d'érosions font une distinction entre deux régimes distincts d'affouillement :

- lorsque le cours d'eau ne charrie pas en amont des ouvrages, l'augmentation locale des contraintes tangentielles donnera une érosion qui s'arrêtera lorsque l'écoulement cessera d'être capable d'arracher des matériaux. Il s'établira alors un équilibre dit "équilibre statique", qui n'est atteint qu'après un temps très long;

- par contre, lorsqu'il y a transport généralisé sur le fond de la rivière, il s'établira un "équilibre dynamique" 
lorsque le débit solide sortant de la cavité est égal à celui qui y entre. Cet équilibre est atteint beaucoup plus rapidement que l'équilibre statique. Dans la majorité des cas pratiques, les rivières en crue ont un charriage généralisé, c'est pourquoi il est plus important de considérer les résultats se rapportant à l'équilibre dynamique.

Comme pour les piles de pont, le paramètre qui intéresse au premier chef le projeteur est la profondeur maximale limite susceptible d'être atteinte pour un ouvrage donné.

III.2.2.2. Erosion due au rétrécissement, cas du rétrécissement long

a) Distinction entre les rétrécissements long et court

Si le rétrécissement est suffisamment long il y a un effet de mise en vitesse de l'eau sur une longueur importante où l'écoulement peut être considéré comme bidimensionnel et uniforme (Fig. 22). Ce phénomène d'érosion, qui se distingue bien des fosses d'affouillement observables en amont des culées, a été étudié analytiquement et expérimentalement.

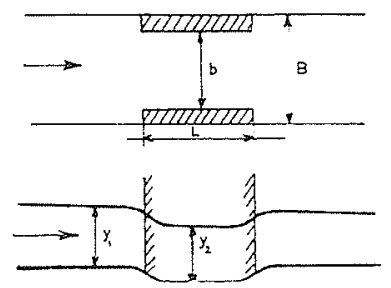

Figure 22 - Ecoulement dans un rétrécissement long.

Par contre, si la longueur $L$ du rétrécissement est petite devant la largeur $b$, le pincement de la veine liquide est immédiatement suivi d'un éclatement; ce phénomène tridimensionnel est très local et ses effets se confondent avec l'affouillement local observable à la tête amont de chaque culée.

La limite généralement adoptée (Komura [19]) pour considérer qu'un rétrécissement est long est $L / b>1$, de sorte que les culées d'un pont ne constituent pas, sauf cas exceptionnels (ponts sur rivières étroites, doubles ponts autoroutiers), des applications de la théorie des rétrécissements longs.

Pour les ponts ordinaires où $L / b<1$, il suffit de considérer les résultats d'affouillements donnés en II.2.2.3, qui intègrent à la fois l'effet local de culée et l'effet de rétrécissement.

\section{b) Erosion dans un rétrécissement long}

Les études théoriques de Staub, Laursen [20] ou Komura [19] et expérimentales de Griffith, Ashida aboutissent à des formules diverses, mais qui font cependant toutes intervenir le rapport de contraction $b / B$.

Dans le cas de l'équilibre dynamique, le rapport des profondeurs dans la section rétrécie $y_{2}$ et dans la section $y_{1}$, peut être prédit par la formule empirique simple

$$
\frac{y_{2}}{y_{1}}=\left(\frac{B}{b}\right)^{2 / 3}
$$

dont il convient, par sécurité, de majorer les résultats de $20 \%$. De telle sorte que la loi à utiliser s'écrit :

$$
\frac{y_{2}}{y_{1}}=1,2\left(\frac{B}{b}\right)^{2 / 3}
$$

Cette loi empirique peut aussi être appliquée à l'équilibre statique.

\section{III.2.2.3. Affouillement local maximal au pied d'une culée}

De nombreuses lois d'affouillement ont été proposées par les chercheurs, leur forme ayant été examinée soit à partir de la théorie du régime, soit à partír des considérations d'analyse dimensionnelle.

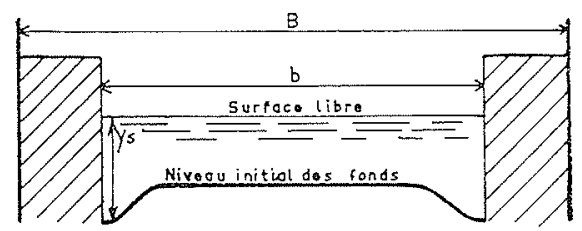

Figure 23 - Affouillement local au pied d'une culée.

Dans le cas de l'équilibre dynamique, nous proposons de retenir la formule empirique de Izard et Bradley [18] qui relie la profondeur extrémale $Y_{s}$ à l'amont de la culée au débit par unité de largeur dans la section rétrécie $q$.

$$
Y_{s}=2,1 q^{2 / 3}
$$

dans le système $M K S$.

Cette relation, établie à partir d'essais sur modèle et vérifiée pour les quelques points nature connus, semble donner de bons résultats pour des sédiments supérieurs à $1,5 \mathrm{~mm}$ environ; pour des sédiments plus fins, il semble qu'il y aurait lieu de majorer de 15 à $20 \%$ les valeurs d'affouillement données par la formule.

Remarque : la formule empirique de Izzard et Bradley ne fait intervenir qu'un seul paramètre pour expliquer l'affouillement. Les divers auteurs qui se sont inté. ressés au problème n'ont pu jusqu'à présent se mettre d'accord sur la liste des paramètres influant sur $Y_{s}$.

Les formules tirées de la théorie du régime sont de la forme

$$
Y_{s}=K q_{1}^{2 / 3} d^{-1 / 6}
$$

avec un coefficient $K$ dépendant seulement de la géométrie de l'obstacle, un débit $q_{1}$ par unité de largeur en amont du pont et un diamètre du sédiment $d$.

Ceci a suggéré à Messieurs Vigoureux et Laraichi [24] la modification suivante de la formule d'Izzard Bradley :

$$
Y_{s}=0,73 \frac{q^{2 / 3}}{d^{1 / 6}}
$$

dans le système $M K S$.

Ce qui permet de mieux décrire les résultats d'essais, de $\mathrm{Da}$ Cunha en particulier, pour les sédiments fins. 


\section{III.2.2.4. Dispositifs de protection}

La protection des culées contre l'érosion a été beaucoup moins étudiés que celle des piles de pont. Da Cunha [17] a essayé d'appliquer aux culées les dispositifs utilisés pour les piles (pilots, collerettes, tapis, panneaux de fond) mais ses essais sont trop fragmentaires pour que des méthodes générales puissent s'en détacher.

Il existe aussi un type de protection spécifique des culées peu répandu dans notre pays, mais qui a été utilisé avec succès à l'étranger, principalement aux USA, il s'agit des "spur-dikes" que l'on peut appeler en français "murs-guideaux" ou "digues-d'approche".

\section{a) Tapis d'enrochements}

Cette méthode éprouvée est la plus utilisée en raison de son efficacité et de sa facilité de mise en ouvre aussi bien à titre préventif que curatif lorsqu'on constate une érosion inattendue.

A notre connaissance des essais systématiques n'ont pas été entrepris visant à définir les dimensions des blocs et du tapis permettant de résister à des conditions hydrauliques données. En l'absence de renseignements, il est possible d'adopter les règles établies pour les piles de pont (se reporter au $\S$ III.2.1.3.c.).

\section{b) Panneaux de fond}

La méthode des panneaux de fonds étudiée par Potapov en URSS à partir de 1932 a été reprise au Laboratoire National d'Hydraulique [25]. Elle consiste à placer au fond du cours d'eau des écrans verticaux faiblement inclinés sur la direction de l'écoulement. Ces panneaux engendrent des courants secondaires qui modifient le transport solide dans le cas de sédiments fins (limons, sables et même graviers) et permettent ainsi de concentrer l'écoulement dans un chenal stable et fixe.

Les panneaux de fond avantageusement utilisés pour les corrections de rivières à courant libre peuvent être appliqués à la fixation du chenal dans le cas d'une rivière instable en plan. Un exemple d'utilisation est celui du pont de Moundou sur la rivière Logone au Tchad, décrit en annexe.

Une technique voisine est celle du "bandalling" utilisée depuis les temps les plus reculés en Asie (Bengale). Elle consiste à disposer des petits rideaux de bambous, en forme de panneaux de surface, qui agissent de façon très analogue à celle des panneaux de fond.

\section{c) Murs guideaux}

a) principe

Les murs guideaux sont constitués par deux digues de forme arrondie, placées en amont des culées et s'y raccordant (fig. 24). Leur but est d'obliger l'écoulement à utiliser toute la section disponible en évitant les décollements; les vitesses et par conséquent les risques d'érosions sont diminués dans la section rétrécie.

Ces ouvrages ont été beaucoup étudiés aux USA par Karaki, Herbich, Scheible (voir les références dans [1]).

Ils peuvent être extrémement utiles pour concentrer, sans dommage, dans le lit mineur une partie du débit du champ d'inondation. Leur emploi est aussi particulièrement judicieux pour les ponts en biais.

\section{B) dimensionnement}

Ces ouvrages étudiés pour l'écoulement le plus fort envisagé, donneront des résultats aussi bons pour tous les débits inférieurs.

C'est une forme arrondie (arc d'ellipse d'excentricité 2,5 à 3) qui semble convenir le mieux. Si les parements de la culée présentent une inclination, les talus du mur-guideau doivent avoir la même pente pour bien épouser la forme de la culée et éviter tout décrochement.

La figure 25 représente une vue type de mur-guideau dont la partie amont est protégée efficement par des enrochements.

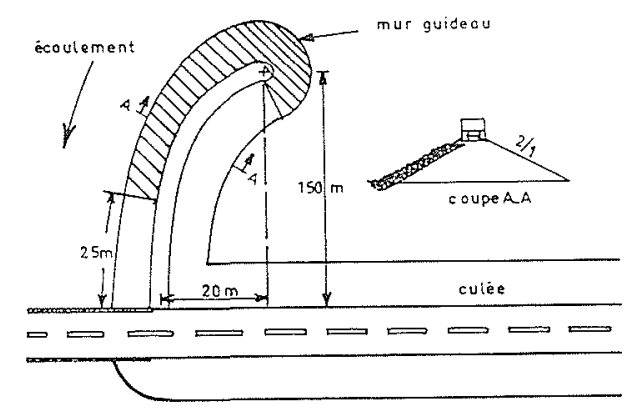

Figure 25 - Dimensionnement d'un mur - guideau type.

L'efficacité d'un mur-guideau est fonction croissante de sa longueur jusqu'à $50 \mathrm{~m}$ environ, au-delà cette influence n'est pas sensible. C'est pourquoi une longueur de $50 \mathrm{~m}$ est généralement recommandée.

Dans le cas particulier des ponts en biais, il est recommandé de traiter de façon particulière la culée aval :

- mur-guideau plus long en amont;

- petit mur-guideau "stub-dike" en aval (Fig. 26).

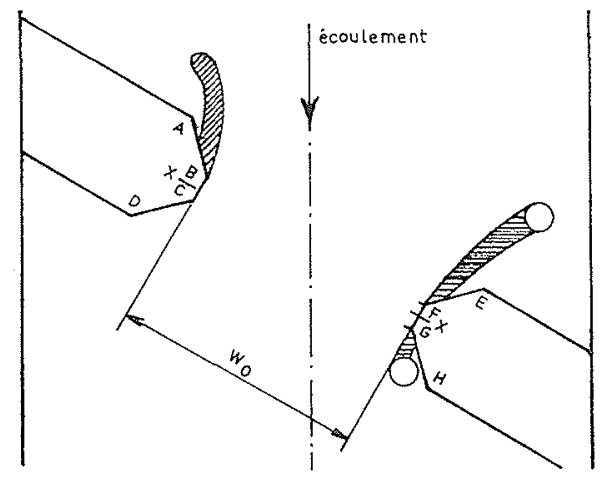

Figure 26 - Murs - guideaux dans le cas d'un pont biais.

Figure 24 - Principe des murs - guideaux. 


\section{III.2.3. Conclusion}

Des formules empiriques approchées permettent de prévoir les affouillements créés par les piles et les culées des ouvrages dans le cas des lits affouillables non cohésifs.

Un choix judicieux des formes de ces structures permet de minimiser ces affouillements. Les dispositifs de protection les plus couramment employés, pour leur faible coût et leur facilité de mise en cuvre, sont les tapis d'enrochements.

Des règles pratiques permettent de dimensionner les matériaux de protection dans le cas des piles; en l'absence de renseignements précis pour les culées, les mêmes lois peuvent être adoptées.

Il convient de remarquer les bons résultats obtenus à l'étranger avec des murs-guideaux qui rendent plus hydrodynamiques les formes de l'entonnement et par là-même améliorent considérablement les performances des ouvrages. Cette méthode serait à développer en France.

Les phénomènes d'affouillement sur sol cohésif restent en domaine très peu exploré [16]; actuellement la seule solution qui s'offre au projecteur pour dimensionner les fondations, dans ce cas, est de suivre les usages (règle de $10 \mathrm{~m}$ du Rhône, du quadruple de marnage, etc.).

\section{Bibliographie}

\section{Chapitre I-Ouvrages traitant du franchissement des cours d'eau en général}

[1] DELLEUR et HERBICH. - Hydraulics of bridged waterways. Final report of Task Committe of ASCE. Report $\mathrm{n}^{\circ} 110$. HEFLD - March 1969. (contient une bibliographie de 90 ouvrages).

[2] NICOLLET et UAN. - Ecoulements permanents à surface libre en lits composés. La Houille blanche $n^{\circ}$ 1, 1979 (p. 21 à 30 ).

\section{Chapitre II-Remous et intéractions écoulement- structure}

[3] LIU, BRADLEY et PLATE. - Backwater effects of piers and abutments. Civil Engeneering section Project. Report CER.57HK L10 Colorado State University October 1957.

[4] Bureau of Public Roads. US Dept of Commerce, Hydraulics of bridge Waterways, Washington 1960.

[5] SKOGERBOE, BARRETT, WALKER et AUSTIN. Comparison of bridge backwater relations, ASCE Hydr. Div., June 1973, p. 921-938.

[6] GALLEZ. - Contribution aux problèmes d'ordre hydraulique relatifs aux piles de pont. Thèse soutenue le 20 juin 1968 devant l'Université de Liège.

[7] MARVAUD et RAMETTE. - Etude des oscillations du plan d'eau dans les passes délimitées par des piles en rivière. VIIIe joumée de l'hydraulique SHF 1964.

[8] APELT et ISAACS. - Bridge piers - Hydrodynamic Force Coefficients. ASCE Hydr. Div. Janv. 1968.

[9] NICOLLET et UAN. - Ecoulements permanents à surface libre en lits composés. La Houille Blanche $n^{\circ} 1,1979$ (p. 21 à 30 ).

[10] VALEMBOIS. - Estimation de la distance de remontée du remous d'un obstacle dans une rivière en crue. La Houille Blanche.

\section{Chapitre III - Erosions}

[11] HENDERSON. - Open Channel Flow. Macmillan 1966.

[12] Comité Français des grands Barrages. - Influence des barrages sur le débit solide et l'évolution du profil en long des cours d'eau. CIGB Madrid 1973. Question 40. Rapport 41.

[13] QUESNEL. - Traité d'Hydraulique Fluviale appliquée. Eyrolles.

[14] CHABERT et ENGELDINGER. - Etude des affouillements autour des piles de pont. LNH Chatou 1956.

[15] BREUSERS, NICOLLET et SHEN. - Local scour around cylindrical piers. Journal of Hydraulic Research., 15 (1977), $\mathrm{n}^{\circ} 3$ (p. 211 à 252).

[16] NICOLLET. - Affouillements autour des piles de pont en milieu cohésif. $16^{e}$ Congrès A.I.R.H. Sao Paulo 1975.

[17] VEIGA DA CUNHA. - Erosoes localizadas junto de obstaculos salientes de margeus. Thèse soutenue à Lisbonne en février 1971.

[18] IZZARD et BRADLEY. - Field verification of model tests on flow through highway brigdes and culverts. 7 th Hydraulics Conference. Iowa 1958.

[19] KOMURA. - Equilibrium depth of scour in long constrictions. Proc. ASCE Hydr. Div., Septembre 1966.

[20] LAURSEN. - An analysis of relief bridge scour around bridged piers and abutments. Proc. ASCE Hydr. Div., May 1963.

[21] SHEN. - Scour near river piers. Rivers Mechanics, Vol. 2 Chap. 23, 1972.

[22] IZBASH et KHALDRE. - Hydraulics of river channel closure. Butterworths 1970.

[23] HANCU. - Sur le calcul des affouillements locaux dans la zone des piles de pont. AIRH 1971, Paris.

[24] LARAICHI et VIGOUREUX. - Règles générales concernant l'implantation des ponts au-dessus des cours d'eau. Travail ENPC 1972, non publié.

[25] CHABERT. - Nouvelle méthode d'aménagement des rivières par utilisation des courants secondaires créés par des panneaux de fond ou de surface. United Nations Congress on the application of science and technology in the interest of underdeveloped regions. Genève 1963.

[26] DAUBERT et LEBRETON. - Etude expérimentale et sur modèle mathématique de quelques aspects des processus d'érosion des lits alluvionnaires en régime permanent et non permanent. $12^{e}$ Congrès de l'A.I.R.H., Septembre 1967, Volume 3, Communication C4.

[27] JEANPIERRE. - L'érosion des fonds, conséquence secondaire de la protection de la plaine d'Alsace par l'endiguement longitudinale du Rhin. $10^{e}$ Joumées de l'Hydraulique, Paris.

\section{Annexe I \\ Historique de quelques cas d'accidents de ponts}

\section{Pont du CD. 11 sur l'Agly}

Sur l'Agly, fleuve côtier torrentiel des Pyrénées Orientales, un pont séculaire protégé par un radier général assurait le passage du CD. 11. Des travaux de recalibrage du fleuve ont été effectués en aval du pont : augmentation de la section d'écoulement par entaillage des berges. Cet aménagement a entraîné une érosion régressive qui provoqua la rupture du radier général et la ruine de l'ouvrage.

\section{Pont de Pulle (Belgique)}

Ce pont permet à l'autoroute Anvers-Liège de traverser le canal Albert; son effrondrement au cours 
d'une nuit a précipité une quarantaine de voitures dans l'eau, entraînant de nombreuses victimes. L'enquête a révélé qu'une pile fondée dans des alluvions avait été complètement déchaussée par le brassage dû aux hélices des chalands automoteurs qui faisaient un point fixe à cet endroit.

\section{Pont du Pouzin sur le Rhône}

$\mathrm{Ce}$ pont a une pile en rivière de forme cylindrique circulaire de 8 mètres de diamètre. Des affouillements de 12 à $13 \mathrm{~m}$ ont été constatés, ils ont été arrêtés par déversement d'enrochements dans la fosse d'érosion de telle sorte qu'une catastrophe a pu être évitée.

\section{Pont de Moundou sur le Logone (Tchad) [A. I]}

Le Logone est une grande rivière africaine caractérisée par une crue annuelle en septembre. Le marnage est d'environ 6 mètres. Le lit du Logone est constitué de sable grossier et d'une proportion assez faible de gravier (10\% environ). Le diamètre médian est d'environ $0,7 \mathrm{~mm}$.

En 1956, a été construit un pont en béton de $350 \mathrm{~m}$ de long engambant le lit mineur et reposant sur 16 piles en rivière. Chaque pile est constituée de deux fûts cylindriques de $1 \mathrm{~m}$ de diamètre espacés de $4 \mathrm{~m}$ entre axes. A l'époque de la construction, le lit mineur était localisé en RG; en RD sur $500 \mathrm{~m}$ environ la traversée du lit majeur a été faite par un remblai assis sur un banc de sable apparemment stabilisé.

Au cours des années, le lit a considérablement évolué, la berge rive droite en amont du pont a été érodée, le banc amont a disparu; le lit mineur s'établissait alors en rive droite; le remblai de la route localisait ainsi, dès 1965 , un chenal profond mettant en danger, d'une part la stabilité de ce remblai, d'autre part, la stabilité des piles RD soumises à un courant traversier très violent et à une érosion intense non prévue dans les calculs de stabilité.

En janvier 1965, à la fin de la crue, les Travaux Publics ont constaté que l'ouvrage était à la limite extrème de stabilité, avec des affouillement de l'ordre de $13 \mathrm{~m}$; les fiches restantes étaient de 5 mètres environ pour les 2 premières piles, alors que les piles avaient été calculées avec une fiche minimum de $8,00 \mathrm{~m}$.

Les travaux entrepris d'urgence ont consisté à protéger par des enrochements les 3 piles RD et la culée. Un chenal a été creusé dans le banc de sable amont pour diriger le plus possible le flot vers l'axe du pont. Une tentative de détoumement du chenal a été faite par des panneaux de fond. Parallèlement des travaux de fixation de la RD ont été entrepris (épis, panneaux) pour arrêter l'évolution du méandre; ces travaux efficaces les premières années se sont avérés insuffisants par la suite.

Seuls des travaux de grande envergure, tels que des murs guideaux amont rejoignant les berges, des épis solidement implantés sur les berges renforcées seraient dans un tel cas efficaces pour fixer le chenal en plan.

\section{Ponts canadiens [A.2]}

Monsieur Neill a étudié 8 cas de ponts dont 3 ont conduit à des accidents : la cause est dûe à l'action de courants obliques sur des piles de forme allongée.

\section{Pont 1.29 sur la Big Sioux River (Iowa) [A. 3]}

Monsieur Anderson rapporte la catastrophe du $1^{\text {er }}$ avril 1962. Les piles de ce pont étaient constituées de deux fûts cylindriques reliés par un voile en béton, ce qui en faisait des piles allongées. Le courant de la crue a frappé ces piles avec une incidence de $25^{\circ}$ environ. Bien que le lit ait été constitué d'un matériau cohésif ( $50 \%$ d'argile et $50 \%$ de sable) une importante érosion a pris naissance; deux piles ont été emportées.

\section{Bibliographie}

[A. 1] Rectification du cours du Logone au pont de Moundou. Document LNH et BCEOM.

[A. 2] NEILL. - Local scour around bridge piers. Research esearch council of Alberta Highways Division. Edmonton, March 1964.

[A. 3] ANDERSON. - The hydraulic design of bridges for river crossings. A case history. 46th Annual meeting of the Highway Research Board. Washington D.C. January 1966.

\section{Annexe II Exemples d'Application}

Exemple 1 : Détermination de l'ouverture d'un pont en lit simple - non affouillable

\section{Données}

Un canal rectiligne prismatique de largeur au plafond $B=100 \mathrm{~m}$, dont les talus ont un fruit $m=3 / 1$ est entièrement revêtu (Fig. 27). Le coefficient de rugosité de Strickler est $S T=80 \mathrm{MKS}$ et la pente moyenne dans la région d'implantation du futur pont est $I=5 \cdot 10^{-4}$.

On veut implanter un pont normalement à la direction de l'écoulement dont les culées de type 2 (voir Fig. 5) sont écartées l'une de l'autre de la distance $L$.

\section{Calcul}

Pour foumir les éléments techniques à l'autorité devant prendre les décisions, le projeteur va rechercher pour diverses ouvertures du pont $L$, quels sont les remous maximaux $h_{1}^{*}$ dus au passage de crues de débit $Q$ par la méthode exposée en II.1.1.2.

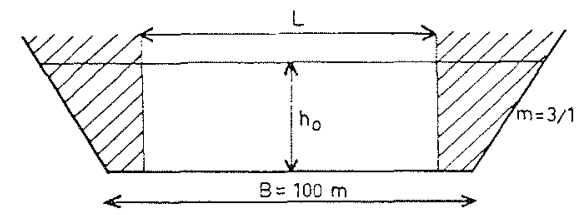

Figure 27 
Le calcul sera fait en supposant l'écoulement uniforme dans la section considérée avant l'implantation du pont.

Si $h_{i}$ est le tirant d'eau avant obstruction assurant le débit $Q$, on en déduit $V_{a}=Q / L h_{i}$.

Si une relation $Q=f(h)$ expérimentale n'est pas connue, on pourra calculer $Q$ par la relation de Strickler :

$$
Q=K S_{i}^{5 / 3} P_{i}^{-2 / 3} I^{1 / 2}
$$

avec

$$
S_{i}=\left(B+m h_{i}\right) h_{i}
$$

$S_{i}=$ section mouillée avant rétrécissement;

et

$$
P_{i}=B+2 h_{i} \sqrt{1+\mathrm{m}^{2}}
$$

$P_{i}=$ périmètre mouillé avant rétrécissement.

Le rapport d'obstruction $M$ est donné par :

$$
M=\frac{Q_{r}}{Q}
$$

en désignant par $Q_{r}$ la fraction du débit qui s'écoulerait dans l'état naturel par le débouché du pont sous le tirant d'eau initial. Donc $M=L h_{i} / S_{i}$.

Sur la figure 5 , on lit la valeur correspondante du coefficient $K_{b}$.

Un calcul donne

$$
V_{a}=\frac{Q}{L h_{i}}, \quad \text { puis } V_{a}^{2} / 2 g
$$

et finalement le remous pour la largeur $L$ et le débit $Q$ par la relation:

$$
h_{1}^{*}=K_{b} \frac{V_{a}^{2}}{2 g}
$$

Après avoir exploré une gamme suffisamment étendue de tirants d'eau $h_{i}$ et de largeurs $L$, on peut établir une série de tableaux du type suivant (Tableau I)

\begin{tabular}{|c|c|c|c|c|c|c|}
\hline \multicolumn{7}{|c|}{ Tableau 1 } \\
\hline $\begin{array}{c}L \\
(\mathrm{~m})\end{array}$ & $\begin{array}{c}h_{f} \\
(\mathrm{~m})\end{array}$ & $\begin{array}{c}Q \\
(\mathrm{~m} / \mathrm{s})\end{array}$ & $M$ & $K_{b}$ & $\frac{V_{a}^{2}}{2 g}$ & $\begin{array}{c}h_{1} \\
(\mathrm{~m})\end{array}$ \\
\hline 90 & 1 & 180 & 0,874 & 0,190 & 0,205 & 0,04 \\
90 & 2 & 578 & 0,849 & 0,232 & 0,526 & 0,12 \\
90 & 3 & 1148 & 0,826 & 0,280 & 0,921 & 0,26 \\
90 & 4 & 1874 & 0,804 & 0,323 & 1,381 & 0,445 \\
\hline
\end{tabular}

Ces résultats permettent de tracer dans le plan $\left(h_{1}^{*}, Q\right.$ ) les courbes $L=$ constante (Fig. 28) et en definitive faire le choix de $L$ correspondant au remous admissible pour un débit donné.

Il est cependant plus pratique de considérer dans le plan $(L, Q)$ la famille des courbes $h_{1}^{*}=$ Cte. Ce graphique donne immédiatement l'ouverture de pont requise pour écouler diverses crues avec un remous donné (Fig. 29).

L'axe des ordonnées peut être gradué en coût de construction de l'ouvrage et celui des abscisses en durée de retour de la crue de débit $Q$.

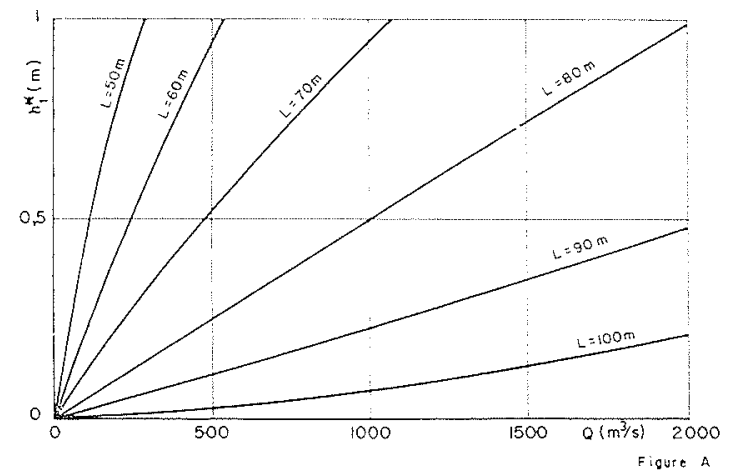

Figure 28 - Courbe donnant la hauteur du remous maximal $h_{1}^{*}$ en fonction du débit de la rivière pour différentes ouverture du pont.

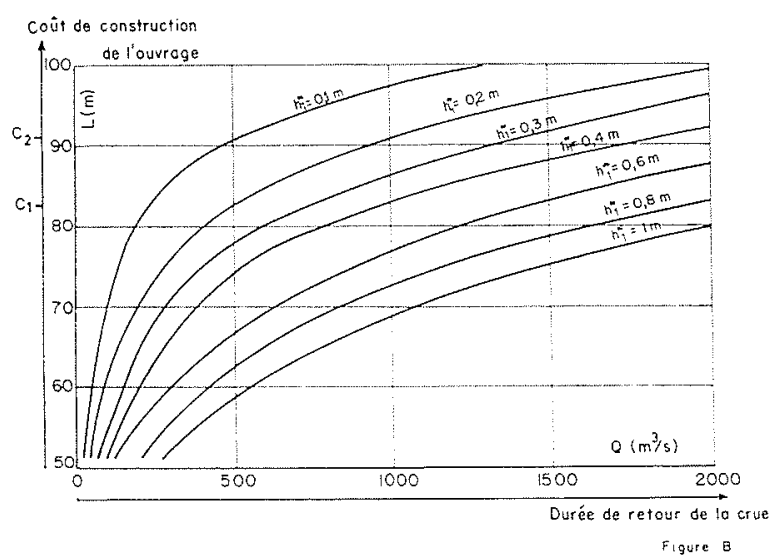

Figure 29 - Courbes donnant l'ouverture du pont en fonction du débit de la rivière pour différentes hauteurs de remous.

Ce document permet à l'autorité responsable de faire un choix. Si par exemple, on peut accepter un remous de $40 \mathrm{~cm}$ pour la crue de projet, dont le débit est de $1000 \mathrm{~m}^{3} / \mathrm{s}$, l'ouverture nécessaire pour le pont est de $82,5 \mathrm{~m}$ environ, d'où le coût de l'ouvrage $C_{1}$. Au contraire, si, on veut, pour cette même crue, limiter le remous à $20 \mathrm{~cm}$, la longueur du pont doit être de $91 \mathrm{~m}$ environ, d'où un coût $C_{2}$. Il est ainsi possible d'apprécier exactement le prix à payer pour limiter le remous du pont.

\section{Remarques}

1) Si l'ouvrage est excentré, ou comporte des piles en rivière, il suffit d'ajouter à $K_{b}$ les termes correctifs donnés par les figures 7 et 8 . Le calcul est exactement le même.

2) $\mathrm{Si}$ le lit du canal est quelconque, une relation $Q=f(Z)$ est nécessaire ( $Z$ désignant la cote de la surface libre). Le processus de calcul du remous $h_{1}^{*}$ reste le même.

Exemple 2 - Détermination de l'ouverture d'un pont et de son ouvrage de décharge en lit majeur

\section{Données}

Le cours d'eau à franchir a son lit mineur assimilable à un rectangle de largeur $200 \mathrm{~m}$; le lit majeur s'étend d'un seul côté sur une largeur de $500 \mathrm{~m}$ (Fig. 30). 


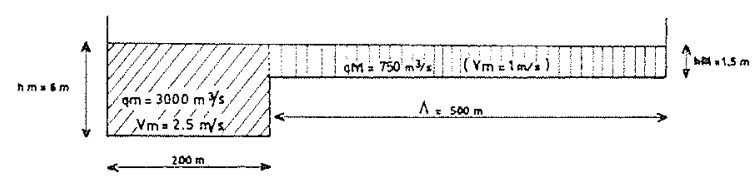

Figure 30 - Section du lit mineur et du lit majeur d'une rivière.

L'étude de la rivière dans l'état naturel a permis de choisir une crue du projet de $3750 \mathrm{~m}^{3} / \mathrm{s}$ s'écoulant pour $80 \%$ par le lit mineur sous la côte indiquée sur la figure 30 .

Les culées des ouvrages seront du type 2 (Fig. 5).

\section{Calculs}

Il s'agit de dimensionner l'ouvrage principal sur le lit mineur et l'ouvrage de décharge en lit majeur. Le principe de calcul consiste à supposer qu'il s'établit une séparation entre les zones d'écoulement de l'ouvrage secondaire telle que les remous soient les mêmes dans ces deux zones.

Pour des débouchés donnés, il s'écoulera par l'ouvrage principal un débit $\left(q_{m}+\alpha q_{M}\right)$ et par l'ouvrage secondaire $q_{M}(1-\alpha)$. Il est possible de tracer pour chacun des ouvrages le remous en fonction de $\alpha: h_{1 m}^{*}(\alpha)$ et $h^{*}(\alpha)$. L'intersection de ces deux courbes donnera la valeur $h_{i}^{*}$ du remous qui s'établit en amont des ouvrages.

Nous donnons ci-dessous, à titre d'exemple, la recherche de la largeur $L^{\prime}$ du débouché en lit majeur, pour un ouvrage d'ouverture égale à la largeur du lit mineur (Fig. 31).

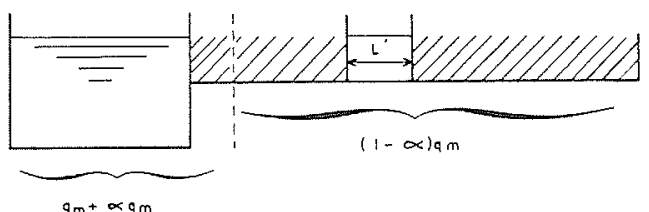

Figure 31

Pour chacun des deux ouvrages, le calcul est conduit selon la méthode présentée dans l'exemple 1.

Ouvrage principal

$$
\begin{aligned}
& q_{1}=q_{m}+\alpha q_{M} \\
& M=\frac{q_{m}}{q_{1}}
\end{aligned}
$$

$K_{b}$ est lu sur la courbe (Fig. 5) (type 2)

$$
\begin{gathered}
V_{a}=\frac{q_{1}}{S_{m}} \text { d'où } \frac{V_{a}^{2}}{2 g} \\
h_{1 m}^{*}=K_{b} \frac{V_{a}^{2}}{2 g}
\end{gathered}
$$

Ouvrage secondaire de largeur $L^{\prime}$ :

$$
\begin{gathered}
q_{2}=(1-\alpha) q_{M} \\
M=\frac{q_{M} \frac{L^{\prime}}{\Lambda}}{q_{2}}
\end{gathered}
$$

$K_{b}$ est lu sur la courbe (Fig. 5) (type 2)

$$
\begin{gathered}
V_{a}^{\prime}=\frac{q_{2}}{L^{\prime} H_{m}} \text { et } \frac{V_{a}^{\prime 2}}{2 g} \\
h_{1 M}^{*}=K_{b}^{\prime} \frac{V_{a}^{\prime 2}}{2 g}
\end{gathered}
$$

Sur la figure 32 sont tracées la courbe $h_{1 m}^{*}(\alpha)$ d'une part et les courbes $h_{1 M}^{*}(\alpha)$ pour $L^{\prime}=50,100$ et $150 \mathrm{~m}$ d'autre part. Les points d'intersection permettent de définir dans le plan $\left(h_{1}^{*}, L^{\prime}\right)$ de la figure 33 la loi de variation du remous en fonction de la largeur $L^{\prime}$ du débouché de l'ouvrage secondaire.

Le même calcul peut être effectué pour diverses largeur de l'ouvrage principal et divers débits jusqu'à obtenir une famille de courbes analogues à celle de la figure 32 de l'exemple 1 , courbes qui permettent de choisir le couple le plus économique des deux ouvrages.

Remarque: Pour ne pas alourdir la présentation de l'exemple, nous avons négligé l'influence de l'excentricité qui n'est pas négligeable pour $L^{\prime}$ petit; en particulier pour $L^{\prime}=0$ (abscence de débouché dans le lit majeur) la prise en compte de l'excentricité fait passer la valeur du remous $h_{1}^{*}$ de 16,5 à $22,5 \mathrm{~cm}$.

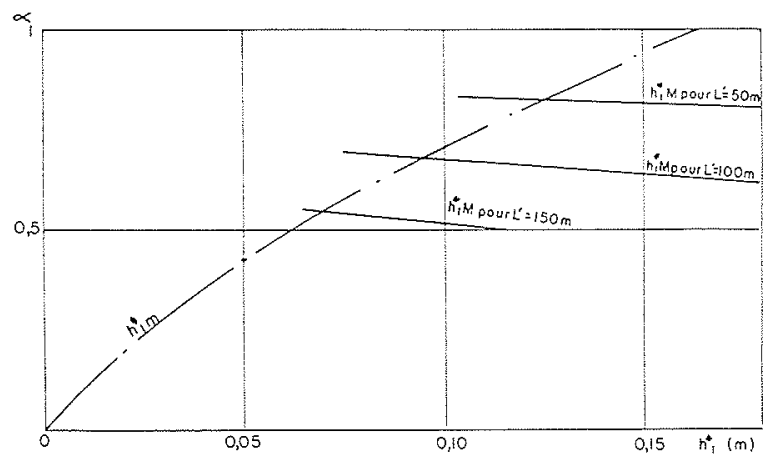

Figure 32 - Calcul de la largeur de l'ouvrage de décharge en lit majeur.

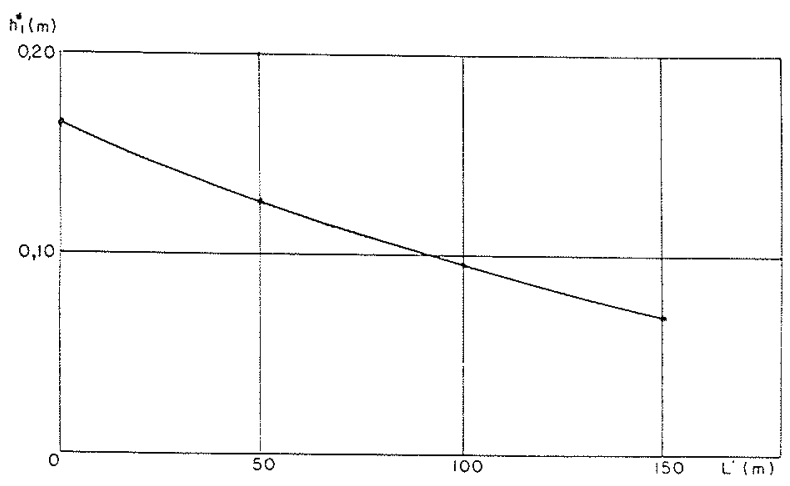

Figure 33 - Courbes donnant la loi de variation du remous $h_{1}^{*}$ en fonction de la largeur $L^{\prime}$ du débouché de l'ouvrage secondaire. 


\section{Exemple 3 - Affouillement au pied de culées}

\section{Données}

Le lit est supposé affouillable

Reprenons les données géométriques de l'exemple 1 pour lequel on a choisi une ouverture de $91 \mathrm{~m}$, le débit de projet étant de $1000 \mathrm{~m}^{3} / \mathrm{s}$. La largeur des culées est de $10 \mathrm{~m}$ (Fig. 34).
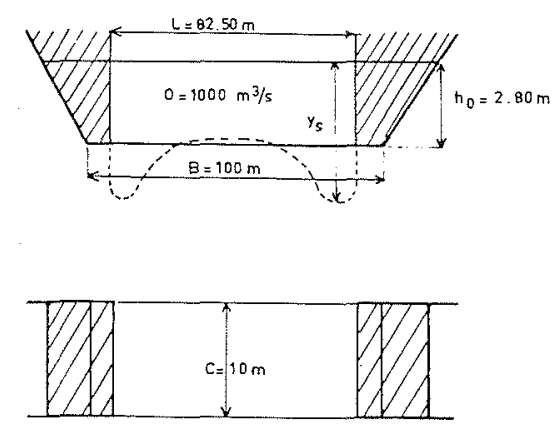

Figure 34

\section{Calcul}

Le rapport $C / L$ de la largeur des culées à l'ouverture du pont est très inférieur à l'unité, on se place donc dans le cas (qui est le plus fréquent) du rétrécissement court.

La profondeur $Y_{s}$ atteinte au pied des culées est données dans ce cas par la formule d'Izzard et Bradley (§ III.2.2.3.) :

$$
Y_{s}=2,1 q^{2 / 3}
$$

en désignant par $q$ le débit par unité de largeur dans la section rétrécie.

On obtient :

$$
q=\frac{1000}{91}=11 \mathrm{~m}^{3} / \mathrm{s} / \mathrm{m}
$$

d'où

$$
Y_{s}=10,4 \mathrm{~m} .
$$

Pour le débit de $1000 \mathrm{~m}^{3} / \mathrm{s}$ la hauteur normale étant de $2,80 \mathrm{~m}$ environ, il y a donc lieu de prévoir des affouillements atteignant $7,60 \mathrm{~m}$ par rapport au niveau moyen du lit, ce qui est extrêmement important et peut remettre en cause le choix de l'ouverture du pont.

La protection peut être réalisée au moyen de blocs d'enrochements capables de résister à une vitesse d'écoulement $V_{c}=2 V_{\max }$ soit ici $V_{c} \simeq 6,72 \mathrm{~m} / \mathrm{s}$. La courbe de la figure 20 traduisant la relation d'Izbash (§ II.2.1.3.) donne des blocs d'environ $1 \mathrm{~m}$ de diamètre.

\section{Exemple 4 - Affouillement au pied des piles}

\section{Données}

Dans le cas du pont envisagé aux exemples 1 et 3 , les piles projetées sont des voiles de béton de $10 \mathrm{~m}$ de longueur et de $1 \mathrm{~m}$ d'épaisseur dont les extrémités sont arrondies. Le matériau mobile a un diamètre moyen compris entre 20 et $25 \mathrm{~mm}$ (Fig. 35).

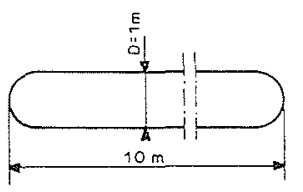

Figure 35 - Affouillement au pied des culées d'un pont.

\section{Calcul}

La valeur de l'affouillement peut être estimée par celle atteinte pour une pile circulaire de diamètre $D=1 \mathrm{~m}$, d'où une valeur maximale $P_{m} \simeq 2 \mathrm{~m}$ par rapport au niveau moyen du lit, ce qui est modeste vis-à-vis de l'affouillement dû aux culées.

La protection par enrochements se détermine par la même méthode que pour les culées et conduit aux mêmes dimensions de blocs. La très grande différence de taille avec les sédiments constitutifs du lit oblige à prévoir un filtre particulièrement soigné dans sa réalisation.

La zone à protéger est représentée sur la figure 36 .

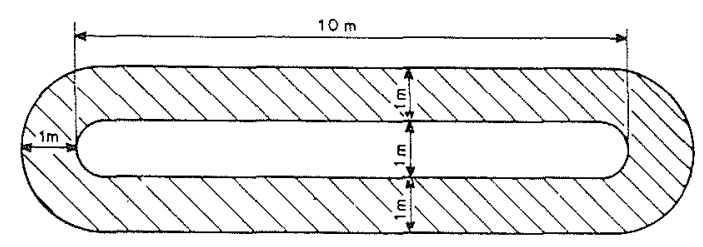

Figure 36 - Section d'une pile projetée. 